\title{
A TRANSPORT EQUATION FOR CONFINED STRUCTURES DERIVED FROM THE BOLTZMANN EQUATION*
}

\author{
CLEMENS HEITZINGER ${ }^{\dagger}$ AND CHRISTIAN RINGHOFER ${ }^{\ddagger}$
}

\begin{abstract}
A system of diffusion-type equations for transport in $3 \mathrm{~d}$ confined structures is derived from the Boltzmann transport equation for charged particles. Transport takes places in confined structures and the scaling in the derivation of the diffusion equation is chosen so that transport and scattering occur in the longitudinal direction and the particles are confined in the two transversal directions. The result are two diffusion-type equations for the concentration and fluxes as functions of position in the longitudinal direction and energy. Entropy estimates are given. The transport coefficients depend on the geometry of the problem that is given by arbitrary harmonic confinement potentials. An important feature of this approach is that the coefficients in the resulting diffusiontype equations are calculated explicitly so that the six position and momentum dimensions of the original 3d Boltzmann equation are reduced to a $2 \mathrm{~d}$ problem. Finally, numerical results are given and discussed. Applications of this work include the simulation of charge transport in nanowires, nanopores, ion channels, and similar structures.
\end{abstract}

Key words. Boltzmann transport equation, many-body system, transport equation, diffusion equation, confined structure, entropy estimate, nanowire, nanopore, nano-structure, ion channel.

AMS subject classifications. 35Q20, 76R50, 82D80.

\section{Introduction}

This paper is concerned with the derivation and analysis of classical sub-band type models for charged particle transport in narrowly confined geometries. The basic idea of sub-band modeling is to treat the transport of particles in the confinement direction on a microscopic level, whereas the transport orthogonal to the confinement direction is treated asymptotically on a much larger time scale.

Sub-band modeling has been employed very successfully in a quantum mechanical setting, where the underlying transport picture is given by the Schrödinger equation. In this case the result is a system of hydrodynamic or diffusion equations for the densities in each sub-band (i.e., the eigenspace of the Schrödinger operator), whose transport coefficients have to be computed by solving eigenvalue problems for the steady-state Schrödinger equation in the confinement direction. We refer the reader to $[5,11]$ for an overview of the physics literature. The hydrodynamic transport picture is then obtained from a large-time asymptotic analysis of the equations for the coefficients in the eigenfunction expansion of the solution of the Schrödinger equation and can be derived rigorously under certain assumptions $[3,2]$.

The present work applies the general sub-band modeling approach to a classical regime, where the underlying transport mechanism is described by a classical kinetic

${ }^{*}$ Received: October 22, 2010; accepted (in revised version): January 16, 2011. Communicated by Pierre Degond.

${ }^{\dagger}$ Department of Applied Mathematics and Theoretical Physics, University of Cambridge, Cambridge CB3 0WA, UK; and Department of Mathematics, University of Vienna, A-1090 Vienna, Austria.

This work was supported by the FWF (Austrian Science Fund) project no. P20871-N13 and by the WWTF (Viennese Science and Technology Fund) project no. MA09-028. This publication is based on work supported by award no. KUK-I1-007-43, funded by the King Abdullah University of Science and Technology (KAUST).

${ }^{\ddagger}$ Department of Mathematics, Arizona State University, Tempe, AZ 85287, USA.

This work was supported by National Science Foundation awards nos. DMS-0604986 and DMS0757309 . 
equation. Therefore the discrete energy levels of the Schrödinger operator are replaced by a continuous energy variable and the final result is a diffusion equation with this energy as an additional independent variable. There are two main differences between the model considered in this paper and the works cited above. First, we consider an essentially arbitrarily complicated geometry. While the papers cited above consider transport in planar thin plates, we consider transport which is confined by an external confinement potential that is produced by an irregular assortment of repulsive wall charges. Second, we consider a regime where the forces produced by these charges are much larger than the external bias, i.e., the force driving the particles through the structure.

The applications in mind here are geometrically complex structures, such as ion channels with a large number of charges on the channel walls $[4,10,12]$, nanowires for sensor applications $[15,13,14,9,7,6,8]$, and nanopores. Consequently, we have to make some simplifying assumptions on the potential outlined below in $\S 2$ as well as on the collision operator to give explicit expressions for the resulting geometry-dependent transport coefficients after a Galerkin discretization.

The rest of this paper is organized as follows. In $\S 3$, a sub-band transport equation for confined structures, which takes the form of a diffusion system, is derived from the Boltzmann transport equation. An entropy estimate is given as well; this is important for the numerics of the equation. In $\S 4$, Galerkin approximations that satisfy the entropy estimate are given. The transport coefficients are first calculated in general form and then explicitly for transport in $3 \mathrm{~d}$ tubes with harmonic (i.e., parabolic) confinement potentials in $\S 5$. Finally, in $\S 6$, numerical solutions are given to show that the calculations can be performed very efficiently and to illustrate the physics of the problem. $\S 7$ concludes the paper.

\section{The transport picture}

The Boltzmann equation for the confined structure, the collision operator, and the dimensionless equation are described in this section.

\subsection{The Boltzmann equation.}

The basic equation. The basic transport picture considered in this paper is given by a Hamiltonian transport term forced by an external potential $V$ and collisions with a background modeled by a Boltzmann-type collision operator. The basic equation is the Boltzmann equation of the form

$$
\partial_{t} f+\{\mathcal{E}, f\}_{X P}+\mathcal{Q}[f]=0 .
$$

Here $f(X, P, t)$ is the kinetic particle density, where $X$ denotes the spatial variable and $P$ denotes the momentum vector. The Hamiltonian transport term is given by the standard commutator, or Poisson bracket,

$$
\{g, f\}_{X P}:=\nabla_{P} g \cdot \nabla_{X} f-\nabla_{X} g \cdot \nabla_{P} f=\nabla_{X} \cdot\left(f \nabla_{P} g\right)-\nabla_{P} \cdot\left(f \nabla_{X} g\right),
$$

and the energy $\mathcal{E}(X, P)$ is given by

$$
\mathcal{E}(X, P):=q_{0} V(X)+\frac{|P|^{2}}{2 m},
$$

where $V(X)$ is the external potential, $|P|^{2} /(2 m)$ the kinetic energy of the particle, $m$ the particle mass, and $q_{0}$ the particle charge. We will use a phenomenological relaxation term, discussed below, for the collision operator $\mathcal{Q}$ in (2.1). 
This paper is concerned with transport in confined geometries, i.e., narrow tubes or thin plates. We therefore split the position and momentum variables into $X=(x, y)$ and $P=(p, q)$, where $x, p \in \mathbb{R}^{d}$ denote position and momentum along the tube or plate and $y, q \in \mathbb{R}^{3-d}$ denote position and momentum in the transversal directions. For a tube $d=1$ holds and for a plate $d=2$ holds.

We consider the case of a narrow geometry, i.e., the extension of the geometry in the $x$-direction is much larger than in the $y$-direction. In this paper the geometry is determined entirely by the potential $V$, which confines the transport to the given geometry and forces the charged particles through the structure at the same time. This is motivated by applications such as ionic channels where confinement is given by fixed repulsive charges on the channel walls. We therefore split the potential $V$ into two parts of the form

$$
q_{0} V(x, y)=K_{B} T\left(V_{0}\left(\frac{x}{L}\right)+V_{1}\left(\frac{x}{L}, \frac{y}{\epsilon L}\right)\right) .
$$

Here $V_{0}$ and $V_{1}$ are dimensionless functions. The parameter $L$ denotes the characteristic length scale of the structure in the $x$-direction, i.e., the transport direction, and $\epsilon L$ is the length scale of the structure in the $y$-direction, i.e., the confinement direction. Hence the dimensionless parameter $\epsilon \ll 1$ is the aspect ratio of the thickness of the tube or plate to its length. $V_{1}$ denotes the confining potential (given by repulsive wall charges) and $V_{0}$ is an external potential driving charged particles through the structure. Here $K_{B}$ denotes the Boltzmann constant and $T$ is the ambient temperature, so that $V_{0}$ and $V_{1}$ are dimensionless.

Remarks.

- The split $V(x, y)=V_{0}(x)+V_{1}(x, y)$ may seem slightly arbitrary at first, since there are of course many ways to decompose a given potential $V(x, y)$. This form is motivated by organizing actual computations, where we want to study the same structure, i.e., the same confining potential $V_{1}$, under different biasing conditions, i.e., for different $V_{0}$. Since the confining potential $V_{1}$ will require extensive preprocessing in the following, whereas the driving potential $V_{0}$ appears directly in the resulting macroscopic model, splitting the potential in this manner proves to be convenient.

- Note that the form (2.3) implies that $\left|\nabla_{y} V\right|=O\left(\frac{1}{\epsilon}\left|\nabla_{x} V\right|\right)$ holds, i.e., the forces confining the particle to the tube or plate are much larger than the external forces driving the particle.

- The confining potential $V_{1}$ models the shape of the geometry that can depend on $x$. For a circular tube or a flat plate, $V_{1}$ could be independent of $x$.

Correspondingly, we split the energy and the Poisson bracket into

$$
\begin{aligned}
\mathcal{E}(X, P) & :=\mathcal{E}_{x}(x, p)+\mathcal{E}_{y}(x, y, q), \\
\{g, f\}_{X P} & =\{g, f\}_{x p}+\{g, f\}_{y q}, \\
\mathcal{E}_{x}(x, p) & :=K_{B} T V_{0}\left(\frac{x}{L}\right)+\frac{|p|^{2}}{2 m}, \\
\mathcal{E}_{y}(x, y, q) & :=K_{B} T V_{1}\left(\frac{x}{L}, \frac{y}{\epsilon L}\right)+\frac{|q|^{2}}{2 m}, \\
\{g, f\}_{x p} & =\nabla_{x} \cdot\left(f \nabla_{p} g\right)-\nabla_{p} \cdot\left(f \nabla_{x} g\right), \\
\{g, f\}_{y q} & =\nabla_{y} \cdot\left(f \nabla_{q} g\right)-\nabla_{q} \cdot\left(f \nabla_{y} g\right) .
\end{aligned}
$$


Using this notation, the Boltzmann Equation (2.1) becomes

$$
\partial_{t} f+\left\{\mathcal{E}_{x}+\mathcal{E}_{y}, f\right\}_{x p}+\left\{\mathcal{E}_{y}, f\right\}_{y q}+\mathcal{Q}[f]=0 .
$$

Collisions. The collision operator $\mathcal{Q}$ in (2.4) models the inelastic interaction of the particle with a background such as water molecules in the case of ionic channels or phonons in the case of transport in a crystal. This interaction will in general result in a gain or loss of the energy $\mathcal{E}(x, y, p, q)$ of the particle. Due to the narrow geometric configuration, these collisions are much more likely to take place in the transport $x$-direction than in the confinement $y$-direction, i.e., the probability of the particle to collide with a background particle with the same $y$-coordinate is much smaller than the probability to collide with a background particle with the same $x$ coordinate. Consequently, we assume that the gain or loss of the cross-directional part $\mathcal{E}_{y}$ of the energy is negligible compared to the change in the transversal part $\mathcal{E}_{x}$. We therefore model collisions by a relaxation operator $\mathcal{Q}$ which locally conserves the cross-directional energy $\mathcal{E}_{y}(x, y, q)$ when averaged over $y$ while relaxing the transversal kinetic energy $\mathcal{E}_{x}(x, p)$ against a Maxwellian distribution according to thermodynamic principles. Hence we define $\mathcal{Q}$ in Equation (2.4) as

$$
\mathcal{Q}[f](x, y, p, q, t):=\frac{1}{\tau}\left(f-M(p) \frac{u_{f}\left(x, \mathcal{E}_{y}(x, y, q), t\right)}{N\left(x, \mathcal{E}_{y}(x, y, q)\right)}\right),
$$

with $M(p)$ being a Maxwellian distribution of the form

$$
M(p):=c \exp \left(-\frac{|p|^{2}}{2 m K_{B} T}\right)
$$

so that

$$
\int M(p) \mathrm{d} p=1
$$

holds. Here $c$ is a normalization constant depending on the dimension $d$ and normalizing the probability distribution $M$. The function $N(x, \eta)$ denotes the $x$-dependent density-of-states function, i.e., the measure of all states with given energy $\mathcal{E}_{y}$ and defined by

$$
N(x, \eta):=\int \delta\left(\mathcal{E}_{y}(x, y, q)-\eta\right) \mathrm{d} y q .
$$

The parameter $\tau$ in (2.5) denotes the relaxation time of the operator. The function $u_{f}(x, \eta, t)$ is defined such that the operator $\mathcal{Q}$ locally conserves the cross-directional energy $\mathcal{E}_{y}$, i.e., the equation

$$
\int \psi\left(\mathcal{E}_{y}(x, y, q)\right) \mathcal{Q}[f](x, y, p, q, t) \mathrm{d} y p q=0 \quad \forall x, t
$$

holds for all test functions $\psi$. For the function $u_{f}$, this implies

$$
u_{f}(x, \eta, t):=\int \delta\left(\mathcal{E}_{y}(x, y, q)-\eta\right) f(x, y, p, q, t) \mathrm{d} y p q .
$$


Summary. The transport picture considered in this paper consists of the interplay between a Hamiltonian operator and a dissipative collision mechanism where, due to the geometry of the structure, the collisions dissipate energy predominately in the transport $x$-direction. As will be seen below, this results in a macroscopic transport picture where the conserved part $\mathcal{E}_{y}$ of the energy appears as an independent variable.

2.2. Scaling and dimensionless formulation. In this section we reformulate the transport problem (2.4) in dimensionless variables, which will enable us to derive an approximate macroscopic (and lower-dimensional) transport equation for a certain asymptotic regime. We scale the spatial variables $x$ and $y$ by $L$ and $\epsilon L$, respectively. Accordingly, we scale the momentum variables $p$ and $q$ by $\left(m q_{0} \bar{V}\right)^{1 / 2}$; we scale time by a characteristic time scale $t_{0}$ to be determined; and we scale the energies $\mathcal{E}_{x}$ and $\mathcal{E}_{y}$ by $q_{0} \bar{V}$. Hence we set

$$
f(x, y, p, q, t):=f_{s}\left(x_{s}, y_{s}, v, w, t_{s}\right)
$$

and

$$
x_{s}:=\frac{x}{L}, \quad y_{s}:=\frac{y}{\epsilon L}, \quad v:=\frac{p}{\sqrt{m K_{B} T}}, \quad w:=\frac{q}{\sqrt{m K_{B} T}}, \quad t_{s}:=\frac{t}{t_{0}} .
$$

We scale the energies by the energy scale $q_{0} \bar{V}$, i.e., $\mathcal{E}_{x}(x, p)=K_{B} T \mathcal{E}_{x s}\left(x_{s}, v\right)$ and $\mathcal{E}_{y}(x, y, q)=K_{B} T \mathcal{E}_{y s}\left(x_{s}, y_{s}, w\right)$, and obtain

$$
\begin{aligned}
\mathcal{E}_{x s}\left(x_{s}, v\right) & =V_{0}\left(x_{s}\right)+\frac{|v|^{2}}{2}, \\
\mathcal{E}_{y s}\left(x_{s}, y_{s}, w\right) & =V_{1}\left(x_{s}, y_{s}\right)+\frac{|w|^{2}}{2} .
\end{aligned}
$$

We scale the collision operator $\mathcal{Q}$ in (2.4) by the collision frequency $1 / \tau$ setting $\mathcal{Q}[f]=$ : $\frac{1}{\tau} \mathcal{Q}_{s}\left[f_{s}\right]$. The scaled and dimensionless version of Equation (2.4) reads

$$
\frac{L \sqrt{m}}{t_{0} \sqrt{K_{B} T}} \partial_{t_{s}} f_{s}+\left\{\mathcal{E}_{x s}+\mathcal{E}_{y s}, f_{s}\right\}_{x_{s} v}+\frac{1}{\epsilon}\left\{\mathcal{E}_{y s}, f_{s}\right\}_{y_{s} w}+\frac{L \sqrt{m}}{\tau \sqrt{K_{B} T}} \mathcal{Q}_{s}\left[f_{s}\right]=0,
$$

where

$$
\begin{aligned}
& \{g, f\}_{x_{s} v}=\nabla_{x_{s}} \cdot\left(f \nabla_{v} g\right)-\nabla_{v} \cdot\left(f \nabla_{x_{s}} g\right), \\
& \{g, f\}_{y_{s} w}=\nabla_{y_{s}} \cdot\left(f \nabla_{w} g\right)-\nabla_{w} \cdot\left(f \nabla_{y_{s}} g\right)
\end{aligned}
$$

and the scaled collision operator $\mathcal{Q}_{s}$ is given by

$$
\begin{aligned}
\mathcal{Q}_{s}\left[f_{s}\right]\left(x_{s}, y_{s}, v, w, t_{s}\right) & =f_{s}-M_{s}(v) \frac{u_{f_{s}}\left(x_{s}, \mathcal{E}_{y s}, t_{s}\right)}{N_{s}\left(x_{s}, \mathcal{E}_{y s}\right)}, \\
u_{f s}\left(x_{s}, \eta, t_{s}\right) & =\int \delta\left(\mathcal{E}_{y s}\left(x_{s}, y_{s}, w\right)-\eta\right) f_{s}\left(x_{s}, y_{s}, v, w, t_{s}\right) \mathrm{d} y_{s} v w \\
M_{s}(v) & =c_{s} \exp \left(-\frac{|v|^{2}}{2}\right), \\
\int M_{s}(v) \mathrm{d} v & =1 .
\end{aligned}
$$


Here $N_{s}$ is the scaled version of the density-of-states function $N$ in (2.6). The scaled version (2.9) of the collision operator $\mathcal{Q}_{s}$ implies the conservation property

$$
\int \psi\left(\mathcal{E}_{y s}\left(x_{s}, y_{s}, w\right)\right) \mathcal{Q}_{s}\left[f_{s}\right]\left(x_{s}, y_{s}, v, w, t_{s}\right) \mathrm{d} y_{s} v w=0 \quad \forall x_{s}, t_{s}
$$

for all test functions $\psi$. We assume that the relaxation time $\tau$ is of the order of the time scale corresponding to transport in the confinement direction $y$, i.e., that $\tau=\tau_{s} \epsilon L \sqrt{m} / \sqrt{K_{B} T}$ and $\tau_{s}=O(1)$ hold.

Finally, we will consider the Boltzmann Equation (2.8) on a diffusion time scale by setting

$$
t_{0}:=\frac{L \sqrt{m}}{\epsilon \sqrt{K_{B} T}}
$$

and obtaining the scaled equation

$$
\epsilon \partial_{t_{s}} f_{s}+\left\{\mathcal{E}_{x s}+\mathcal{E}_{y s}, f_{s}\right\}_{x_{s} v}+\frac{1}{\epsilon}\left\{\mathcal{E}_{y s}, f_{s}\right\}_{y_{s} w}+\frac{1}{\epsilon \tau_{s}} \mathcal{Q}_{s}\left[f_{s}\right]=0 .
$$

From here on we drop the subscript $s$ for the scaled variables for notational simplicity.

2.3. Summary. We summarize the assumptions describing the physical regime under consideration.

- Transport occurs in a narrow, irregular pipe or plate $(d=1$ or $d=2$, respectively) with a geometric aspect ratio $\epsilon$.

- The confining forces are much larger than the external forces that drive the particles through the structure and therefore $\left|\nabla_{x} V\right|=O\left(\epsilon\left|\nabla_{y} V\right|\right)$ holds.

- Collisions with the background conserve the cross-directional energy $\mathcal{E}_{y}$ while dissipating the energy $\mathcal{E}_{x}$ in the transport direction $x$.

- Collisions occur frequently on the time scale of transport in the $x$-direction which, for a given potential, is given by $L \sqrt{m} / \sqrt{K_{B} T}$.

In the following, a diffusion equation on a large time scale $t_{0}=L \sqrt{m} /\left(\epsilon \sqrt{K_{B} T}\right)$ will be derived from the transport Equation (2.11) using a standard Chapman-Enskog approach. The final result of the asymptotic procedure is a conservation law of the form

$$
\partial_{t} \rho(x, \eta, t)+\nabla_{x} \cdot F^{x}(x, \eta, t)+\partial_{\eta} F^{\eta}(x, \eta, t)=0,
$$

where the additional variable $\eta$ denotes the energy $\mathcal{E}_{y}$ in $(2.7)$ on the macroscopic level. While the large-time asymptotic analysis is quite straightforward, the actual computation of the transport coefficients is quite involved due to the asymptotic conservation properties of the effective collision operator $\left\{\mathcal{E}_{y}, f\right\}_{y w}+\mathcal{Q}[f]$ and requires special considerations and simplifying assumptions (see $\S 5$ ) to make the procedure computationally efficient. In the end, the transport coefficients are given explicitly.

\section{The transport equation for confined structures}

In this section we derive a large-time approximation to the dimensionless Equation (2.11) using a standard Chapman-Enskog type procedure. The result is a diffusion equation for the density

$$
\rho(x, \eta, t):=\int \delta\left(\mathcal{E}_{y}(x, y, w)-\eta\right) f(x, y, v, w, t) \mathrm{d} y v w,
$$


where the cross-directional energy $\mathcal{E}_{y}$ appears as the additional variable $\eta$. We also derive an entropy estimate showing that the resulting diffusion problem is actually well-posed.

3.1. The Chapman-Enskog expansion. First, we observe that the linear relaxation operator $\mathcal{Q}$ in (2.9) is a projection operator. We define the projection operator $\mathcal{P}$ by

$$
\begin{aligned}
\mathcal{P}[f](x, y, v, w, t) & :=\frac{\rho_{f}\left(x, \mathcal{E}_{y}(x, y, w), t\right)}{N\left(x, \mathcal{E}_{y}(x, y, w)\right)} M(v), \\
N(x, \eta) & =\int \delta\left(\mathcal{E}_{y}(x, y, w)-\eta\right) \mathrm{d} y w, \\
\int \psi\left(\mathcal{E}_{y}\right)(\mathcal{P}[f]-f)(x, y, v, w, t) \mathrm{d} y v w & =0 \quad \forall x, t
\end{aligned}
$$

for all test functions $\psi(\eta)$. Here $M(v)$ denotes the scaled Maxwellian in (2.9) and $N(x, \eta)$ is the scaled density-of-states function. As before the definition (3.1) implies that, for given $f$, the function $\rho_{f}(x, \eta, t)$ is given by

$$
\rho_{f}(x, \eta, t):=\int \delta\left(\mathcal{E}_{y}(x, y, w)-\eta\right) f(x, y, v, w, t) \mathrm{d} y v w,
$$

and a straightforward calculation yields that $\mathcal{P}$ is indeed a projection operator, i.e., that $\mathcal{P}^{2}=\mathcal{P}$ holds.

Hence the relaxation operator $\mathcal{Q}$ in (2.11) is a projection operator of the form $\mathcal{Q}=$ $\mathcal{I}-\mathcal{P}$ with $\mathcal{I}$ being the identity operator. The projection $\mathcal{P}$ projects onto the linear manifold of functions which are multiples of the Maxwellian $M(v)$ and depend on $y$ and $w$ only through the energy $\mathcal{E}_{x}(x, y, w)$. The Chapman-Enskog expansion consists of deriving the asymptotic form of the Boltzmann equation in local coordinates on this manifold. To this end, we split the density function $f(x, y, v, w, t)$ into $f=f_{0}+\epsilon f_{1}$ with

$$
\begin{aligned}
f_{0}(x, y, v, w, t) & :=\mathcal{P}[f](x, y, v, w, t), \\
f_{1}(x, y, v, w, t) & :=\frac{1}{\epsilon}(\mathcal{I}-\mathcal{P})[f](x, y, v, w, t) .
\end{aligned}
$$

We also split the evolution Equation (2.11) by applying the projections $\mathcal{P}$ and $\mathcal{I}-\mathcal{P}$.

Before doing so, we observe that the Poisson bracket $\left\{\mathcal{E}_{y}, .\right\}_{y w}$ is a directional derivative in a direction orthogonal to the kernel manifold of $\mathcal{Q}$. To compute the projection $\mathcal{P}\left[\left\{\mathcal{E}_{y}, f\right\}_{y w}\right]$ for a general function $f$, we compute

$$
\begin{aligned}
& \rho_{\left\{\mathcal{E}_{y}, f\right\}_{y w}}(x, \eta, t)=\int \delta\left(\mathcal{E}_{y}(x, y, w)-\eta\right)\left\{\mathcal{E}_{y}, f\right\}_{y w} \mathrm{~d} y v w \\
&=\int\left\{\delta\left(\mathcal{E}_{y}(x, y, w)-\eta\right), \mathcal{E}_{y}\right\}_{y w} f \mathrm{~d} y v w=0
\end{aligned}
$$

according to (3.2). The above identity holds because of the cyclicity of the commutator trace, i.e., $\int a\{b, c\}_{y w} \mathrm{~d} y w=\int\{a, b\}_{y w} c \mathrm{~d} y w$; moreover, the definition (2.2) of the Poisson bracket implies that $\left\{\mathcal{E}_{y}, \phi\right\}_{y w}=0$ holds for any function $\phi$ which depends on $y$ and $w$ only through the energy $\mathcal{E}_{y}$. Because of (3.3) and the definition of $\mathcal{P}$, we therefore have the identity

$$
\mathcal{P}\left[\left\{\mathcal{E}_{y}, f\right\}_{y w}\right]=0 \quad \forall f
$$


Hence applying the projections $\mathcal{P}$ and $\mathcal{I}-\mathcal{P}$ to the Boltzmann Equation (2.11) yields the system

$$
\begin{array}{r}
\epsilon \partial_{t} f_{0}+\mathcal{P}\left[\left\{\mathcal{E}_{x}+\mathcal{E}_{y}, f_{0}+\epsilon f_{1}\right\}_{x v}\right]=0, \\
\epsilon^{2} \partial_{t} f_{1}+(\mathcal{I}-\mathcal{P})\left[\left\{\mathcal{E}_{x}+\mathcal{E}_{y}, f_{0}+\epsilon f_{1}\right\}_{x v}\right]+\frac{1}{\epsilon}\left\{\mathcal{E}_{y}, f_{0}+\epsilon f_{1}\right\}_{y w}+\frac{1}{\tau} f_{1}=0 .
\end{array}
$$

The system (3.4) can be simplified further using again the algebraic properties of the Poisson bracket and the projection operator $\mathcal{P}$. First, we note that the function $f_{0}$ as well as the energy $\mathcal{E}_{x}+\mathcal{E}_{y}$ are even functions of the velocity $v$; therefore the commutator $\left\{\mathcal{E}_{x}+\mathcal{E}_{y}, f_{0}\right\}_{x v}$ is an odd function of $v$, the integral with respect to $v$ in the definition (3.1) and (3.2) of the projection operator vanishes, and thus $\mathcal{P}\left[\left\{\mathcal{E}_{x}+\mathcal{E}_{y}, f_{0}\right\}_{x v}\right]=0$ holds. Second, $f_{0}$ depends on $y$ and $w$ only through the energy $\mathcal{E}_{y}$; therefore, and because of the definition (2.2) of the commutator, we have $\left\{\mathcal{E}_{y}, f_{0}\right\}_{y w}=0$. Using these two properties in the system (3.4), we obtain

$$
\begin{array}{r}
\epsilon \partial_{t} f_{0}+\mathcal{P}\left[\left\{\mathcal{E}_{x}+\mathcal{E}_{y}, \epsilon f_{1}\right\}_{x v}\right]=0, \\
\epsilon^{2} \partial_{t} f_{1}+\left\{\mathcal{E}_{x}+\mathcal{E}_{y}, f_{0}+\epsilon f_{1}\right\}_{x v}-\mathcal{P}\left[\left\{\mathcal{E}_{x}+\mathcal{E}_{y}, \epsilon f_{1}\right\}_{x v}\right]+\frac{1}{\epsilon}\left\{\mathcal{E}_{y}, \epsilon f_{1}\right\}_{y w}+\frac{1}{\tau} f_{1}=0 .
\end{array}
$$

Equation (3.5a) gives the evolution on the kernel manifold of the operator $\mathcal{Q}$ and Equation (3.5b) gives the evolution on the orthogonal complement. The macroscopic approximation is now obtained by formally dropping the $O(\epsilon)$ terms in (3.5b), i.e., the term $\epsilon f_{1}$ will stay small for all time assuming that we start on the kernel manifold (i.e., $\epsilon f_{1}=0$ holds at $t=0$ ). This yields the system

$$
\begin{array}{r}
\partial_{t} f_{0}+\mathcal{P}\left[\left\{\mathcal{E}_{x}+\mathcal{E}_{y}, f_{1}\right\}_{x v}\right]=0, \\
\left\{\mathcal{E}_{x}+\mathcal{E}_{y}, f_{0}\right\}_{x v}+\left\{\mathcal{E}_{y}, f_{1}\right\}_{y w}+\frac{1}{\tau} f_{1}=0 .
\end{array}
$$

3.2. The conservation law. Using the definition of the projection operator $\mathcal{P}$, Equation (3.6a) can be written as a conservation law for the density $\rho_{f}(x, \eta, t)$. To see this, we integrate (3.6a) against the test function $\psi=\delta\left(\mathcal{E}_{y}(x, y, w)-\eta\right)$ with respect to $y, v$, and $w$ and use the definition (3.1) and (3.2) of the projection operator to find

$$
\partial_{t} \rho_{f_{0}}(x, \eta, t)+\Phi(x, \eta, t)=0
$$

where

$$
\Phi(x, \eta, t):=\int \delta\left(\mathcal{E}_{y}(x, y, w)-\eta\right)\left\{\mathcal{E}_{x}+\mathcal{E}_{y}, f_{1}\right\}_{x v} \mathrm{~d} y v w
$$

This constitutes a conservation law, since we have

$$
\int \Phi(x, \eta, t) \mathrm{d} x \eta=\int\left\{\mathcal{E}_{x}+\mathcal{E}_{y}, f_{1}\right\}_{x v} \mathrm{~d} x y v w=0
$$


and therefore we should be able to write $\Phi$ in divergence form as $\Phi=\nabla_{x} \cdot F^{x}+\partial_{\eta} F^{\eta}$. Integrating $\Phi(x, \eta, t)$ against a test function $\psi(x)$ yields

$$
\begin{aligned}
& \int \psi(x) \Phi(x, \eta, t) \mathrm{d} x \\
= & \int \psi(x) \delta\left(\mathcal{E}_{y}-\eta\right)\left\{\mathcal{E}_{x}+\mathcal{E}_{y}, f_{1}\right\}_{x v} \mathrm{~d} x y v w \\
= & \int\left\{(x) \delta\left(\mathcal{E}_{y}-\eta\right), \mathcal{E}_{x}+\mathcal{E}_{y}\right\}_{x v} f_{1} \mathrm{~d} x y v w \\
= & \int\left(\nabla_{v}\left(\psi(x) \delta\left(\mathcal{E}_{y}-\eta\right)\right) \cdot \nabla_{x}\left(\mathcal{E}_{x}+\mathcal{E}_{y}\right)-\nabla_{x}\left(\psi(x) \delta\left(\mathcal{E}_{y}-\eta\right)\right) \cdot \nabla_{v}\left(\mathcal{E}_{x}+\mathcal{E}_{y}\right)\right) f_{1} \mathrm{~d} x y v w \\
= & -\int \nabla_{x}\left(\psi(x) \delta\left(\mathcal{E}_{y}-\eta\right)\right) \cdot \nabla_{v}\left(\mathcal{E}_{x}+\mathcal{E}_{y}\right) f_{1} \mathrm{~d} x y v w \\
= & -\int \delta\left(\mathcal{E}_{y}-\eta\right) \nabla_{x} \psi(x) \cdot v f_{1} \mathrm{~d} x y v w-\int \psi(x) \delta^{\prime}\left(\mathcal{E}_{y}-\eta\right) \nabla_{x} V_{1} \cdot v f_{1} \mathrm{~d} x y v w \\
= & \int \psi(x) \nabla_{x} \cdot\left(\delta\left(\mathcal{E}_{y}-\eta\right) v f_{1}\right) \mathrm{d} x y v w+\int \psi(x) \partial_{\eta}\left(\delta\left(\mathcal{E}_{y}-\eta\right)\left(\nabla_{x} V_{1} \cdot v\right) f_{1}\right) \mathrm{d} x y v w .
\end{aligned}
$$

This constitutes the weak (in $x$ ) definition of the fluxes $F^{x}$ and $F^{\eta}$. The conservation law is given by

$$
\partial_{t} \rho_{f_{0}}(x, \eta, t)+\nabla_{x} \cdot F^{x}+\partial_{\eta} F^{\eta}=0
$$

where the fluxes are

$$
\begin{aligned}
& F^{x}(x, \eta, t):=\int \delta\left(\mathcal{E}_{y}-\eta\right) v f_{1} \mathrm{~d} y v w \\
& F^{\eta}(x, \eta, t):=\int \delta\left(\mathcal{E}_{y}-\eta\right)\left(\nabla_{x} V_{1} \cdot v\right) f_{1} \mathrm{~d} y v w .
\end{aligned}
$$

\section{Remarks.}

- Equation (3.8) constitutes a conservation law for the mesoscopic fluid density $\rho_{f_{0}}$, which still depends on the free energy $\eta=\mathcal{E}_{y}$. Thus the resulting model is similar to a SHE (spherical-harmonics expansion) model [1] for the Boltzmann equation except that only a part of the energy appears as an independent variable.

- The challenge is of course to compute the fluxes $F^{x}$ and $F^{\eta}$ in (3.8), i.e., to compute the density $f_{1}$ from $(3.6 \mathrm{~b})$ for a given $f_{0}$ of the form $f_{0}(x, y, v, w, t)=$ $M(v) \rho_{f_{0}}\left(x, \mathcal{E}_{y}, t\right) / N\left(x, \mathcal{E}_{y}\right)$.

- It is easy to see that the resulting mesoscopic equation for $\rho_{f_{0}}$ will contain second-order partial derivatives with respect to $x$ and $\eta$. This raises the question of well-posedness of Equation (3.8). We will address this issue below by providing an entropy estimate for the system.

3.3. An entropy estimate. The goal of this section is twofold. The first goal is to show that the system (3.6) is well-posed. To that end, we will show that there is a convex functional of the density $\rho$-an entropy - which decays in time. To make the system (3.6) amenable to actual computations, it will be necessary to solve (3.6b) approximately via a form of series expansion. The second goal of this section is therefore to characterize the structure of the system (3.6) in such a way that we 
can formulate a Galerkin approximation to (3.6b) which retains the same stability properties.

We start from the system (3.6) and express the kinetic density $f_{0}$ in terms of the mesoscopic density $\rho_{f_{0}}$ as $f_{0}(x, y, v, w, t)=\rho_{f_{0}}\left(x, \mathcal{E}_{y}, t\right) M(v) / N\left(x, \mathcal{E}_{y}\right)$. (For notational simplicity, we drop the subscript $f_{0}$ of the mesoscopic density $\rho_{f_{0}}$ from here on.) We write the system (3.6) in terms of linear operators $\mathcal{L}_{1}$ and $\mathcal{L}_{2}$ as

$$
\begin{array}{r}
\partial_{t} \rho(x, \eta, t)+\mathcal{L}_{1}\left[f_{1}\right](x, \eta, t)=0, \\
\mathcal{L}_{2}[\rho](x, y, v, w, t)+\left\{\mathcal{E}_{y}, f_{1}\right\}_{y w}+\frac{1}{\tau} f_{1}=0,
\end{array}
$$

with the operators $\mathcal{L}_{1}$ and $\mathcal{L}_{2}$ defined by

$$
\begin{aligned}
\mathcal{L}_{1}\left[f_{1}\right](x, \eta, t) & :=\int \delta\left(\mathcal{E}_{y}(x, y, w)-\eta\right)\left\{\mathcal{E}_{x}+\mathcal{E}_{y}, f_{1}\right\}_{x v} \mathrm{~d} y v w, \\
\mathcal{L}_{2}[\rho](x, y, v, w, t) & :=\left\{\mathcal{E}_{x}+\mathcal{E}_{y}, M(v) \frac{\rho\left(x, \mathcal{E}_{y}, t\right)}{N\left(x, \mathcal{E}_{y}\right)}\right\}_{x v} .
\end{aligned}
$$

The operator $\mathcal{L}_{1}$ maps functions of the form $f_{1}(x, y, v, w, t)$ to functions of the form $\rho(x, \eta, t)$, whereas the operator $\mathcal{L}_{2}$ operates in the opposite direction. The operator $\mathcal{L}_{2}$ is related to the adjoint of the operator $\mathcal{L}_{1}$ by Lemma 3.1 below. Since we will use a combination of trigonometric basis functions for the Galerkin procedure in the next section and it will be notationally convenient to write these basis functions as complex exponentials, we define the adjoint of an operator over the complex numbers; the solutions $\rho$ and $f_{1}$ are of course real functions.

Let $\mathcal{L}_{1}^{\text {adj }}$ denote the adjoint of $\mathcal{L}_{1}$ with respect to the $L^{2}$ inner product, i.e.,

$$
\int \rho(x, \eta, t)^{*} \mathcal{L}_{1}[f](x, \eta, t) \mathrm{d} x \eta=\int \mathcal{L}_{1}^{\text {adj }}[\rho](x, y, w, t)^{*} f(x, y, v, w, t) \mathrm{d} x y v w
$$

holds, where ${ }^{*}$ denotes the complex conjugate.

LEMma 3.1 (adjoint property). Let $\mathcal{L}_{1}^{\text {adj }}$ denote the adjoint of $\mathcal{L}_{1}$. Then the operators $\mathcal{L}_{1}$ and $\mathcal{L}_{2}$ in (3.10) are related by the equation

$$
\mathcal{L}_{2}[\rho](x, y, v, w, t)=-c \mathrm{e}^{-\mathcal{E}_{x}-\mathcal{E}_{y}} \mathcal{L}_{1}^{\text {adj }}\left[\frac{\mathrm{e}^{V_{0}(x)+\eta} \rho(x, \eta, t)}{N(x, \eta)}\right](x, y, v, w, t) .
$$

Here the constant $c$ denotes the normalization constant of the Maxwellian so that $M(v)=c \exp \left(-|v|^{2} / 2\right)$ holds. Furthermore the identity

$$
\mathfrak{R e}\left(\int \mathrm{e}^{\mathcal{E}_{x}+\mathcal{E}_{y}} f^{*}\left\{\mathcal{E}_{y}, f\right\}_{y w} \mathrm{~d} y w\right)=0 \quad \forall x
$$

holds for all complex functions $f(y, w)$, where $\mathfrak{R e}$ denotes the real part.

The proof is deferred to Appendix A.

Using Lemma 3.1, the system (3.9) becomes

$$
\begin{array}{r}
\partial_{t} \rho(x, \eta, t)+\mathcal{L}_{1}\left[f_{1}\right](x, \eta, t)=0, \\
-c \mathrm{e}^{-\mathcal{E}_{x}-\mathcal{E}_{y}} \mathcal{L}_{1}^{\text {adj }}\left[\frac{\mathrm{e}^{V_{0}(x)+\eta} \rho(x, \eta, t)}{N(x, \eta)}\right](x, y, v, w, t)+\left\{\mathcal{E}_{y}, f_{1}\right\}_{y w}+\frac{1}{\tau} f_{1}=0 .
\end{array}
$$


The significance of the form (3.13) of the system is twofold. First, it allows for the straightforward proof of an entropy estimate below and therefore guarantees that the resulting diffusion system is well-posed. Second, as will be seen in the next section, expressing $f_{1}$ in terms of $\rho$ from (3.13b) is a nontrivial matter, and will have to be done numerically in general. In order for this numerical approximation (and the resulting diffusion equation) to obey the same entropy estimate, we will use the form (3.13).

As a consequence of Lemma 3.1, we immediately obtain the entropy estimate.

Proposition 3.2 (entropy estimate). Solutions $\left(\rho, f_{1}\right)$ of the system (3.13) satisfy the entropy estimate

$$
\frac{1}{2} \partial_{t} \int \frac{\mathrm{e}^{V_{0}(x)+\eta}}{N(x, \eta)}|\rho(x, \eta, t)|^{2} \mathrm{~d} x \eta=-\frac{1}{c \tau} \int \mathrm{e}^{\mathcal{E}_{x}+\mathcal{E}_{y}}\left|f_{1}\right|^{2} \mathrm{~d} x y v w \leq 0 .
$$

Proof. Integrating (3.13a) against $\mathrm{e}^{V_{0}(x)+\eta} \rho(x, \eta, t)^{*} / N(x, \eta)$ with respect to $x$ and $\eta$, integrating (3.13b) against $\frac{1}{c} e^{\mathcal{E}_{x}+\mathcal{E}_{y}} f_{1}^{*}$ with respect to $x, y, v$, and $w$, and adding yields

$$
\begin{aligned}
\int \frac{\mathrm{e}^{V_{0}+\eta} \rho^{*}}{N} \partial_{t} \rho(x, \eta, t) \mathrm{d} x \eta+\int \frac{\mathrm{e}^{V_{0}+\eta} \rho^{*}}{N} \mathcal{L}_{1}\left[f_{1}\right] \mathrm{d} x \eta-\int f_{1}^{*} \mathcal{L}_{1}^{\text {adj }}\left[\frac{\mathrm{e}^{V_{0}+\eta} \rho}{N}\right] \mathrm{d} x y v w \\
+\frac{1}{c} \int \mathrm{e}^{\mathcal{E}_{x}+\mathcal{E}_{y}} f_{1}^{*}\left\{\mathcal{E}_{y}, f_{1}\right\}_{y w} \mathrm{~d} x y v w+\frac{1}{c \tau} \int \mathrm{e}^{\mathcal{E}_{x}+\mathcal{E}_{y}}\left|f_{1}\right|^{2} \mathrm{~d} x y v w=0 .
\end{aligned}
$$

Taking the real part and using Lemma 3.1 concludes the proof.

\section{Galerkin approximations}

Equation (3.13b) must be solved approximately by a form of series-expansion method for practical computations. To prepare the system for this approximation procedure, we will perform two steps below, while guaranteeing that the structure and the stability estimate given by Lemma 3.1 and Proposition 3.2 remain unchanged.

4.1. Eliminating the lateral velocity $v$. We observe that the dependence of the operator $\mathcal{L}_{2}$ in $(3.10 \mathrm{~b})$ on the velocity $v$ takes the form of a multiple of the function $M(v) v$, i.e.,

$$
\mathcal{L}_{2}[\rho](x, y, v, w, t)=M(v) v \cdot\left(\nabla_{x}\left(\frac{\rho\left(x, \mathcal{E}_{y}, t\right)}{N\left(x, \mathcal{E}_{y}\right)}\right)+\frac{\rho\left(x, \mathcal{E}_{y}, t\right)}{N\left(x, \mathcal{E}_{y}\right)} \nabla_{x}\left(V_{0}+V_{1}\right)\right)
$$

holds. Since the Poisson bracket $\left\{\mathcal{E}_{y}, f_{1}\right\}_{y w}$ does not operate on the velocity component $v$, Equation (3.9b) allows a solution of the form

$$
f_{1}(x, y, v, w, t)=M(v) v \cdot g(x, y, w, t) .
$$

Here the function $g(x, y, w, t) \in \mathbb{R}^{d}$ is vector valued for the case of a plate and scalar in the case of a tube. Correspondingly, we define the operators $\Lambda_{1}$ and $\Lambda_{2}$ as

$$
\begin{aligned}
\Lambda_{1}[g](x, \eta, t) & :=\mathcal{L}_{1}[M(v) v \cdot g](x, \eta, t), \\
\mathcal{L}_{2}[\rho](x, y, v, w, t) & =M(v) v \cdot \Lambda_{2}[\rho](x, y, w, t), \\
\Lambda_{2}[\rho](x, y, w, t) & :=\nabla_{x}\left(\frac{\rho\left(x, \mathcal{E}_{y}, t\right)}{N\left(x, \mathcal{E}_{y}\right)}\right)+\frac{\rho\left(x, \mathcal{E}_{y}, t\right)}{N\left(x, \mathcal{E}_{y}\right)} \nabla_{x}\left(V_{0}+V_{1}\right)
\end{aligned}
$$


and the system (3.9) becomes

$$
\begin{aligned}
\partial_{t} \rho(x, \eta, t)+\Lambda_{1}[g](x, \eta, t) & =0, \\
\Lambda_{2}[\rho](x, y, w, t)+\left\{\mathcal{E}_{y}, g\right\}_{y w}+\frac{1}{\tau} g & =0 .
\end{aligned}
$$

Here $\Lambda_{2}[\rho]$ and $g$ are $d$-dimensional vector functions and the commutator $\left\{\mathcal{E}_{y}, g\right\}_{y w}$ acts componentwise on $g$. Correspondingly, we define the adjoint of the operator $\Lambda_{1}$ (over the complex numbers) by

$$
\int \rho(x, \eta, t)^{*} \Lambda_{1}[g](x, \eta, t) \mathrm{d} x \eta=\int \Lambda_{1}^{\operatorname{adj}}[\rho](x, \eta, t)^{H} g(x, y, w, t) \mathrm{d} x \eta \quad \forall \rho, g,
$$

where $g^{H}$ denotes the Hermitian conjugate $g^{H}=\left(g^{T}\right)^{*}$.

Lemma 4.1 (adjoint property). The operator $\Lambda_{1}$ is of the form

$$
\Lambda_{1}[g](x, \eta, t)=\int \nabla_{x} \cdot\left(\delta\left(\mathcal{E}_{y}-\eta\right) g(x, y, w, t)\right)+\partial_{\eta}\left(\delta\left(\mathcal{E}_{y}-\eta\right) \nabla_{x} V_{1}(x, y) \cdot g\right) \mathrm{d} y w,
$$

and the operator $\Lambda_{2}$ is given in terms of the adjoint of $\Lambda_{1}$ by

$$
\Lambda_{2}[\rho](x, y, w, t)=-\mathrm{e}^{-V_{0}-\mathcal{E}_{y}} \Lambda_{1}^{\text {adj }}\left[\mathrm{e}^{V_{0}+\eta} \frac{\rho(x, \eta, t)}{N(x, \eta)}\right](x, y, w, t) .
$$

Furthermore the identity

$$
\mathfrak{R e}\left(\int \mathrm{e}^{\mathcal{E}_{x}+\mathcal{E}_{y}} g^{H}\left\{\mathcal{E}_{y}, g\right\}_{y w} \mathrm{~d} y w\right)=0 \quad \forall x
$$

holds for all complex functions $g(y, w)$.

The proof is deferred to Appendix B.

Thus, using Lemma 4.1, the system (4.2) becomes

$$
\begin{aligned}
\partial_{t} \rho(x, \eta, t)+\Lambda_{1}[g](x, \eta, t) & =0, \\
-\mathrm{e}^{-V_{0}-\mathcal{E}_{y}} \Lambda_{1}^{\text {adj }}\left[\mathrm{e}^{V_{0}+\eta} \frac{\rho(x, \eta, t)}{N(x, \eta)}\right](x, y, w, t)+\left\{\mathcal{E}_{y}, g\right\}_{y w}+\frac{1}{\tau} g(x, y, w, t) & =0 .
\end{aligned}
$$

4.2. Energy and angle variables. In order to obtain a closed equation for the mesoscopic density $\rho(x, \eta, t)$, Equation $(4.6 \mathrm{~b})$ has to be inverted for $g$ in terms of $\rho$. For a general confinement potential $V_{1}(x, y)$, this must be done approximatively. This approximation will take the form of a series expansion, i.e., Galerkin solution, of (4.6b). To facilitate this procedure, we assume the existence of a one-to-one variable transformation in $(y, w)$ space mapping the $(6-2 d)$-dimensional vector $(y, w)$ into the scalar energy $u=\mathcal{E}_{y}(x, y, w)$ and a $(5-2 d)$-dimensional angular variable $\theta$. This means we assume the existence of an $x$-dependent variable transformation $(u, \theta)=\Gamma(x, y, w)$ and its functional inverse $(y, w)=\Omega(x, u, \theta)$ satisfying

$$
\mathcal{E}_{y}(x, \Omega(x, u, \theta))=u, \quad \Gamma(x, \Omega(x, u, \theta))=(u, \theta), \quad \Omega(x, \Gamma(x, y, w))=(y, w)
$$

for all $x \in \mathbb{R}^{d}, y, w \in \mathbb{R}^{3-d}, u \in \mathbb{R}$, and $\theta \in \mathbb{R}^{5-2 d}$. The Galerkin approximation will then take the form of a series expansion in the angular variable $\theta$. Employing the transformation $(y, w)=\Omega(x, u, \theta)$, we define the operator $\mathcal{A}$ as

$$
\begin{aligned}
g_{1}(x, u, \theta, t) & :=g(x, \Omega(x, u, \theta), t), \\
\mathcal{A}\left[g_{1}\right](x, \eta, t) & :=\Lambda_{1}[g](x, \eta, t) .
\end{aligned}
$$


To compute the system (4.6) after the transformation $(y, w)=\Omega(x, u, \theta)$, we have to compute the appropriate transforms of the operators $\Lambda_{1}, \Lambda_{1}^{\text {adj }}$, and $\left\{\mathcal{E}_{y}, g\right\}_{y w}$.

We compute the transformed operator $\mathcal{A}$ first. To this end, it will be necessary to split the transformation $\Omega$ into

$$
\left(\begin{array}{c}
y \\
w
\end{array}\right)=\Omega(x, u, \theta)=\left(\begin{array}{c}
\Omega_{y}(x, u, \theta) \\
\Omega_{w}(x, u, \theta)
\end{array}\right) .
$$

We also define the Jacobian matrices

$$
\partial \Omega(x, u, \theta):=\frac{\partial(y, w)}{\partial(u, \theta)}, \quad \partial \Gamma(x, y, w):=\frac{\partial(u, \theta)}{\partial(y, w)}
$$

and the volume element $\sigma(x, u, \theta):=|\operatorname{det}(\partial \Omega(x, u, \theta))|$. The following lemma gives the operator $\mathcal{A}$ and its adjoint $\mathcal{A}^{\text {adj }}$.

Lemma 4.2. The operator $\mathcal{A}$ defined in (4.7) is given by

$$
\mathcal{A}\left[g_{1}\right](x, \eta, t)=\int \nabla_{x} \cdot\left(\sigma g_{1}(x, \eta, \theta, t)\right)+\partial_{\eta}\left(\sigma(x, \eta, \theta) \nabla_{1} V_{1}\left(x, \Omega_{y}(x, \eta, \theta)\right) \cdot g_{1}\right) \mathrm{d} \theta .
$$

Its adjoint, defined by $\int g_{1}^{H} \mathcal{A}^{\text {adj }}[\rho] \mathrm{d} x u \theta=\int \mathcal{A}\left[g_{1}\right]^{*} \rho \mathrm{d} x \eta$ for all $g_{1}$ and $\rho$, is given by

$$
\mathcal{A}^{\text {adj }}[\rho](x, u, \theta, t)=\sigma(x, u, \theta) \Lambda_{1}^{\text {adj }}[\rho](x, \Omega(x, u, \theta), t) .
$$

Proof. Integrating $\mathcal{A}\left[g_{1}\right]$ against a test function $\phi(x, \eta)$ yields

$$
\begin{aligned}
& \int \phi(x, \eta) \mathcal{A}\left[g_{1}\right](x, \eta, t) \mathrm{d} x \eta \\
= & -\int \delta\left(\mathcal{E}_{y}-\eta\right)\left(\nabla_{x} \phi(x, \eta) \cdot g(x, y, w)+\partial_{\eta} \phi(x, \eta) \nabla_{x} V_{1}(x, y) \cdot g\right) \mathrm{d} x y w \eta
\end{aligned}
$$

after a calculation similar to (3.7) and using the identity $\int v^{T} v M(v) \mathrm{d} v=I$ (see the beginning of Appendix B for this identity). Substituting $(y, w)=\Omega(x, u, \theta)$ and $\mathrm{d} y w=$ $\sigma(x, u, \theta) \mathrm{d} u \theta$ in the integral yields

$$
\begin{aligned}
& \int \phi(x, \eta) \mathcal{A}\left[g_{1}\right] \mathrm{d} x \eta=-\int \delta(u-\eta) \\
& \quad \cdot\left(\nabla_{x} \phi(x, \eta) \cdot g_{1}(x, u, \theta, t)+\partial_{\eta} \phi(x, \eta) \nabla_{1} V_{1}\left(x, \Omega_{y}(x, u, \theta)\right) \cdot g_{1}\right) \sigma(x, u, \theta) \mathrm{d} x u \theta \eta,
\end{aligned}
$$

where we denote the partial gradient of $V_{1}$ with respect to the first variable by $\nabla_{1} V_{1}$. Since a zero energy $u=\mathcal{E}_{y}=0$ will correspond to infinitely many angles $\theta, \sigma(x, 0, \theta)=0$ will hold giving the usual singularity at the origin of spherical-type variable transformations. Therefore integrating out the variable $u$ and integrating by parts with respect to $x$ and $\eta$ gives the strong formulation of the operator $\mathcal{A}$ in (4.8).

To prove Equation (4.9), we compute

$$
\int \rho^{*} \Lambda_{1}[g](x, \eta, t) \mathrm{d} x \eta=\int \rho^{*} \mathcal{A}\left[g_{1}\right](x, \eta, t) \mathrm{d} x \eta=\int \Lambda_{1}^{\text {adj }}[\rho]^{H} g(x, y, w, t) \mathrm{d} x y w
$$

for all $\rho, g$, and $g_{1}$ and obtain the identity

$$
\int \rho^{*} \mathcal{A}\left[g_{1}\right](x, \eta, t) \mathrm{d} x \eta=\int \Lambda_{1}^{\mathrm{adj}}[\rho](x, y, w, t)^{H} g_{1}(x, \Gamma(x, y, w), t) \mathrm{d} x y w .
$$


Substituting $(y, w)=\Omega(x, u, \theta)$ in the last integral gives

$$
\int \mathcal{A}^{\text {adj }}[\rho]^{H} g_{1}(x, u, \theta, t) \mathrm{d} x u \theta=\int \sigma(x, u, \theta) \Lambda_{1}^{\text {adj }}[\rho](x, \Omega(x, u, \theta), t)^{H} g_{1}(x, u, \theta, t) \mathrm{d} x u \theta
$$

for all $\rho$ and $g_{1}$, which yields Equation (4.9).

It remains to compute the transformation of the Poisson bracket $\left\{\mathcal{E}_{y}, g\right\}_{y w}$ under the variable transformation $(y, w)=\Omega(x, u, \theta)$. Since for a function $\phi$ of the form $\phi(x, y, w)=\phi\left(x, \mathcal{E}_{y}(x, y, w)\right)$ the Poisson bracket satisfies $\left\{\mathcal{E}_{y}, \phi\right\}_{y w}=0$, the commutator $\left\{\mathcal{E}_{y}, .\right\}_{y w}$ is essentially a directional derivative along the equipotential surface $\mathcal{E}_{y}(x, y, w)=u$. Therefore, after the transformation $(y, w)=\Omega(x, u, \theta)$, the transformed Poisson bracket contains only derivatives with respect to the angular variable $\theta$. This fact is expressed in Lemma 4.3 below. But first we partition the Jacobian matrices $\partial \Omega$ and $\partial \Gamma$ into

$$
\begin{aligned}
& \partial \Omega(x, u, \theta)=\frac{\partial(y, w)}{\partial(u, \theta)}=\left(\begin{array}{ll}
\frac{\partial y}{\partial u} & \frac{\partial y}{\partial \theta} \\
\frac{\partial w}{\partial u} & \frac{\partial w}{\partial \theta}
\end{array}\right)=:\left(\begin{array}{ll}
\omega_{11} & \omega_{12} \\
\omega_{21} & \omega_{22}
\end{array}\right), \\
& \partial \Gamma(x, y, w)=\frac{\partial(u, \theta)}{\partial(y, w)}=\left(\begin{array}{ll}
\frac{\partial u}{\partial y} & \frac{\partial u}{\partial w} \\
\frac{\partial \theta}{\partial y} & \frac{\partial \theta}{\partial w}
\end{array}\right)=:\left(\begin{array}{ll}
\gamma_{11} & \gamma_{12} \\
\gamma_{21} & \gamma_{22}
\end{array}\right) .
\end{aligned}
$$

LEMmA 4.3. Under the transformation $(y, w)=\Omega(x, u, \theta)$ with $g(x, y, w, t)=$ $g_{1}(x, u, \theta, t)$, the Poisson bracket $\left\{\mathcal{E}_{y}, g\right\}_{\text {yw }}$ becomes

$$
\left\{\mathcal{E}_{y}, g\right\}_{y w}(x, \Omega(x, u, \theta), t)=\left(S \cdot \nabla_{\theta}\right) g_{1}(x, u, \theta, t),
$$

with the $(5-2 d)$-dimensional vector $S$ given by

$$
S(x, u, \theta):=\left(\gamma_{21} \gamma_{12}^{T}-\gamma_{22} \gamma_{11}^{T}\right)(x, \Omega(x, u, \theta)) .
$$

Furthermore, the identity

$$
\mathfrak{R e}\left(\int \sigma(x, u, \theta) \mathrm{e}^{\mathcal{E}_{x}+u} g_{1}(x, u, \theta, t)^{H}\left(S \cdot \nabla_{\theta}\right) g_{1}(x, u, \theta, t) \mathrm{d} u \theta\right)=0 \quad \forall x
$$

holds for all vector functions $g_{1}$.

The proof is deferred to Appendix C.

Remarks.

- $S$ is a vector in $\mathbb{R}^{5-2 d}$, i.e., it has the same dimension as the angle $\theta$, and therefore the operator $S \cdot \nabla_{\theta}$ is scalar and acts componentwise on the vector $g_{1}$ in (4.10).

- Lemma 4.3 expresses the fact that the commutator $\left\{\mathcal{E}_{y},\right\}_{y w}$ is in fact a directional derivative. If $g(x, y, w, t)$ is of the form $g=g\left(x, \mathcal{E}_{y}(x, y, w), t\right)$, then $g_{1}$ is of the form $g_{1}(x, u, \theta, t)=g_{1}(x, u, t)$ and $\left(S \cdot \nabla_{\theta}\right) g_{1}=0$ holds.

Lemmas 4.2 and 4.3 allow us to write the system (4.6) from the previous section in terms of the transformed variables $(u, \theta)=\Gamma(x, y, w)$. Hence we obtain the system

$$
\begin{aligned}
\partial_{t} \rho(x, \eta, t)+\mathcal{A}\left[g_{1}\right](x, \eta, t) & =0, \\
-\mathrm{e}^{-V_{0}-u} \mathcal{A}^{\text {adj }}\left[\mathrm{e}^{V_{0}+\eta} \frac{\rho(x, \eta, t)}{N(x, \eta)}\right](x, u, \theta, t)+\sigma\left(S \cdot \nabla_{\theta}\right) g_{1}+\frac{\sigma}{\tau} g_{1}(x, u, \theta, t) & =0,
\end{aligned}
$$




$$
\begin{aligned}
\mathcal{A}\left[g_{1}\right](x, \eta, t) & =\int \nabla_{x} \cdot\left(\sigma g_{1}(x, \eta, \theta, t)\right)+\partial_{\eta}\left(\sigma(x, \eta, \theta) \nabla_{1} V_{1}\left(x, \Omega_{y}(x, \eta, \theta)\right) \cdot g_{1}\right) \mathrm{d} \theta \\
S(x, u, \theta) & =\left(\gamma_{21} \gamma_{12}^{T}-\gamma_{22} \gamma_{11}^{T}\right)(x, \Omega(x, u, \theta)) \\
\sigma(x, u, \theta) & =|\operatorname{det}(\partial \Omega(x, u, \theta))| .
\end{aligned}
$$

Remark: Since the operator $\mathcal{A}$ in (4.13c) is such that the function $g_{1}$ is evaluated only at $u=\eta$, no confusion is produced by renaming the independent variable from $u$ to $\eta$ in Equations (4.13b), (4.13d), and (4.13e) from here on.

4.3. Series expansions. In order to obtain a closed system for the density $\rho$, Equation (4.13b) must be solved for $g_{1}$ in terms of $\rho$ and the result must be substituted for $g_{1}$ in (4.13a). This inversion cannot be performed exactly in general, but must be approximated by a series expansion. The point of Sections 4.1 and 4.2 was to transform the system into a form such that the resulting approximate equation still satisfies the entropy estimate of Proposition 3.2 in $\S 3.3$ because of the adjoint structure in Equation (4.13).

We choose an orthonormal system of basis functions $\kappa_{K}(\theta)$ with $K \in \mathcal{K}$ satisfying

$$
\int \kappa_{K}(\theta)^{*} \kappa_{K^{\prime}}(\theta) \mathrm{d} \theta=\delta_{K K^{\prime}}
$$

where $K$ is a multiindex varying in a $(5-2 d)$-dimensional index set $\mathcal{K}$. We expand the function $g_{1}$ in (4.13) into the basis functions by setting

$$
g_{1}(x, \eta, \theta, t)=\sum_{K \in \mathcal{K}} \kappa_{K}(\theta) G_{K}(x, \eta, t) .
$$

Since we will use a combination of trigonometric functions for the basis functions $\kappa_{K}$, we allow for complex valued basis functions $\kappa_{K}$ and therefore the coefficients $G_{K}(x, \eta, t)$ are $d$-dimensional complex valued coefficient vectors. (Of course, the complex exponentials sum so that $g_{1}$ and $\rho$ are real functions.) Equation (4.13b) is then replaced by

$$
\begin{aligned}
-\int \kappa_{K}(\theta)^{*} \mathrm{e}^{-V_{0}-\eta} \mathcal{A}^{\text {adj }}\left[\mathrm{e}^{V_{0}+\eta} \frac{\rho(x, \eta, t)}{N(x, \eta)}\right](x, \eta, \theta, t) \mathrm{d} \theta & \\
& +\int \kappa_{K}(\theta)^{*}\left(\sigma(x, \eta, \theta)\left(S \cdot \nabla_{\theta}\right) g_{1}+\frac{\sigma}{\tau} g_{1}(x, \eta, \theta, t)\right) \mathrm{d} \theta=0 \quad \forall K \in \mathcal{K} .
\end{aligned}
$$

We remark that, because of the construction via adjoint operators, the entropy estimate from $\S 3.3$ carries over directly to the approximate system regardless of the choice of basis functions. Multiplying Equation (4.15) by $\mathrm{e}^{V_{0}+\eta} G_{K}^{H}$, summing over the index $K$, integrating with respect to $x$ and $\eta$, taking real parts, and using Equation (4.12) yields

$$
-\mathfrak{R e}\left(\int \mathrm{e}^{V_{0}+\eta} \frac{\rho(x, \eta, t)^{*}}{N(x, \eta)} \mathcal{A}\left[g_{1}\right](x, \eta, t) \mathrm{d} x \eta\right)+\int \frac{\sigma}{\tau} \mathrm{e}^{V_{0}+\eta}\left|g_{1}(x, \eta, \theta, t)\right|^{2} \mathrm{~d} x \eta \theta=0 .
$$

Integrating (4.13a) against $\mathrm{e}^{V_{0}+\eta} \rho(x, \eta, t)^{*} / N(x, \eta)$ and adding the two equations then yields again the entropy estimate

$$
\frac{1}{2} \partial_{t} \int \mathrm{e}^{V_{0}+\eta} \frac{|\rho(x, \eta, t)|^{2}}{N(x, \eta)} \mathrm{d} x \eta=-\int \frac{\sigma}{\tau} \mathrm{e}^{V_{0}+\eta}\left|g_{1}(x, \eta, \theta, t)\right|^{2} \mathrm{~d} x \eta \theta \leq 0
$$


regardless of the choice of basis functions and the number of terms used in the Galerkin approximation.

The rest of this section is devoted to computing the flux terms $F^{x}$ and $F^{\eta}$ given by $\nabla_{x} \cdot F^{x}+\partial_{\eta} F^{\eta}=\mathcal{A}\left[g_{1}\right]$ in Equation (4.13) which, because of Equation (4.8) in Lemma 4.2 , are of the form

$$
\begin{aligned}
& F^{x}=\int \sigma g_{1}(x, \eta, \theta, t) \mathrm{d} \theta, \\
& F^{\eta}=\int \sigma \nabla_{1} V_{1}\left(x, \Omega_{y}(x, \eta, \theta)\right) \cdot g_{1} \mathrm{~d} \theta
\end{aligned}
$$

for a given confining potential $V_{1}$ and a given variable transformation $\Omega$. Applying the operator $\mathcal{A}$ in (4.13c) to a function $g_{1}$ of the form $g_{1}(x, u, \theta, t)=\kappa_{K}(\theta) G(x, \eta, t)$ yields

$$
\mathcal{A}\left[\kappa_{K} G\right](x, \eta, t)=\nabla_{x} \cdot\left(a_{K}(x, \eta) G(x, \eta, t)\right)+\partial_{\eta}\left(A_{K}(x, \eta) \cdot G(x, \eta, t)\right),
$$

where the scalars $a_{K}$ and the $d$-dimensional vectors $A_{K}$ are given by

$$
\begin{aligned}
& a_{K}(x, \eta):=\int \kappa_{K}(\theta) \sigma(x, \eta, \theta) \mathrm{d} \theta, \\
& A_{K}(x, \eta):=\int \kappa_{K}(\theta) \sigma(x, \eta, \theta) \nabla_{1} V_{1}\left(x, \Omega_{y}(x, \eta, \theta)\right) \mathrm{d} \theta .
\end{aligned}
$$

After using the Galerkin approximation, the conservation law (4.13a) becomes

$$
\partial_{t} \rho(x, \eta, t)+\sum_{K \in \mathcal{K}} \nabla_{x} \cdot\left(a_{K}(x, \eta) G_{K}(x, \eta, t)\right)+\partial_{\eta}\left(A_{K}(x, \eta) \cdot G_{K}(x, \eta, t)\right)=0 .
$$

We remark that the variable transformation $(y, w)=\Omega(x, \eta, \theta)$ will necessarily be singular at $\eta=0$, since a zero energy will correspond to any possible angle. Therefore, as is always the case in a transformation onto spherical coordinates, $\sigma(x, 0, \theta)=0$ will hold and therefore the coefficients $a_{K}$ and $A_{K}$ will vanish at $\eta=0$ as well.

We now turn to computing the matrix elements in Equation (4.15). We first compute the term

$$
R_{K}(x, \eta, t):=\int \kappa_{K}(\theta)^{*} \mathrm{e}^{-V_{0}(x)-\eta} \mathcal{A}^{\text {adj }}\left[\mathrm{e}^{V_{0}+\eta} \frac{\rho(x, \eta, t)}{N(x, \eta)}\right](x, \eta, \theta, t) \mathrm{d} \theta .
$$

Integrating $R_{K}$ against a vector valued test function $\mathrm{e}^{V_{0}+\eta} \phi(x, \eta)$ yields

$$
\begin{aligned}
& \int\left(\mathrm{e}^{V_{0}+\eta} \phi(x, \eta)\right)^{H} R_{K}(x, \eta, t) \mathrm{d} x \eta \\
= & \int\left(\kappa_{K}(\theta) \phi(x, \eta)\right)^{H} \mathcal{A}^{\text {adj }}\left[\mathrm{e}^{V_{0}+\eta} \frac{\rho(x, \eta, t)}{N(x, \eta)}\right](x, \eta, \theta, t) \mathrm{d} x \eta \theta \\
= & \int \mathcal{A}\left[\kappa_{K}(\theta) \phi(x, \eta)\right]^{*} \mathrm{e}^{V_{0}+\eta} \frac{\rho(x, \eta, t)}{N(x, \eta)} \mathrm{d} x \eta \theta \\
= & \int\left(\nabla_{x} \cdot\left(a_{K}(x, \eta) \phi(x, \eta)\right)+\partial_{\eta}\left(A_{K}(x, \eta) \cdot \phi(x, \eta)\right)\right)^{*} \mathrm{e}^{V_{0}+\eta} \frac{\rho(x, \eta, t)}{N(x, \eta)} \mathrm{d} x \eta \\
= & \int-a_{K}(x, \eta)^{*} \phi(x, \eta)^{H} \nabla_{x}\left(\mathrm{e}^{V_{0}+\eta} \frac{\rho}{N}\right)-A_{K}(x, \eta)^{*} \phi(x, \eta)^{H} \partial_{\eta}\left(\mathrm{e}^{V_{0}+\eta} \frac{\rho}{N}\right) \mathrm{d} x \eta .
\end{aligned}
$$


This implies

$$
\mathrm{e}^{V_{0}+\eta} R_{K}(x, \eta, t)=-a_{K}(x, \eta)^{*} \nabla_{x}\left(\mathrm{e}^{V_{0}+\eta} \frac{\rho}{N}\right)-A_{K}(x, \eta)^{*} \partial_{\eta}\left(\mathrm{e}^{V_{0}+\eta} \frac{\rho}{N}\right) .
$$

The second term in Equation (4.15) does not operate on the variables $x$ and $\eta$. Therefore inserting the expansion (4.14) for $g_{1}$ into (4.15) results just in multiplication with an $(x, \eta)$-dependent matrix $C$. With the definition

$$
C_{K K^{\prime}}(x, \eta):=\int \kappa_{K}(\theta)^{*}\left(\sigma(x, \eta, \theta)\left(S \cdot \nabla_{\theta}\right) \kappa_{K^{\prime}}(\theta)+\frac{\sigma}{\tau} \kappa_{K^{\prime}}(\theta)\right) \mathrm{d} \theta,
$$

Equation (4.15) becomes

$$
\begin{aligned}
a_{K}(x, \eta)^{*} \mathrm{e}^{-V_{0}} \nabla_{x}\left(\mathrm{e}^{V_{0}} \frac{\rho}{N}\right)+A_{K}(x, \eta)^{*} \mathrm{e}^{-\eta} \partial_{\eta}\left(\mathrm{e}^{\eta} \frac{\rho}{N}\right) & \\
& +\sum_{K^{\prime} \in \mathcal{K}} C_{K K^{\prime}}(x, \eta) G_{K^{\prime}}(x, \eta, t)=0 \quad \forall K \in \mathcal{K} .
\end{aligned}
$$

Summary. For a given confinement potential $V_{1}(x, y)$ and for a given variable transformation $(y, w)=\Omega(x, \eta, \theta)=\left(\Omega_{y}, \Omega_{w}\right)$ and $(\eta, \theta)=\Gamma(x, y, w)$, we have to compute the coefficients $a_{K}(x, \eta), A_{K}(x, \eta)$, and $C_{K K^{\prime}}(x, \eta)$ given by

$$
\begin{aligned}
a_{K}(x, \eta) & =\int \kappa_{K}(\theta) \sigma(x, \eta, \theta) \mathrm{d} \theta, \\
A_{K}(x, \eta) & =\int \kappa_{K}(\theta) \sigma(x, \eta, \theta) \nabla_{1} V_{1}\left(x, \Omega_{y}(x, \eta, \theta)\right) \mathrm{d} \theta, \\
C_{K K^{\prime}}(x, \eta) & =\int \kappa_{K}(\theta)^{*} \sigma(x, \eta, \theta)\left(\left(S \cdot \nabla_{\theta}\right) \kappa_{K^{\prime}}(\theta)+\frac{1}{\tau} \kappa_{K^{\prime}}(\theta)\right) \mathrm{d} \theta, \\
S(x, \eta, \theta) & =\left(\gamma_{21} \gamma_{12}^{T}-\gamma_{22} \gamma_{11}^{T}\right)(x, \Omega(x, \eta, \theta)), \\
\sigma(x, \eta, \theta) & =|\operatorname{det}(\partial \Omega(x, \eta, \theta))|
\end{aligned}
$$

and solve the coupled system $(4.17),(4.18)$ for the density $\rho(x, \eta, t)$ and the $d$ dimensional expansion coefficients $G_{K}(x, \eta, t), K \in \mathcal{K}$. Using the inverse of the matrix $C$ (assuming it exists) to express the coefficients $G_{K}$ in (4.18) in terms of the density $\rho$ in (4.17) yields the conservation law

$$
\partial_{t} \rho(x, \eta, t)+\nabla_{x} \cdot F^{x}(x, \eta, t)+\partial_{\eta} F^{\eta}(x, \eta, t)=0,
$$

where the $d$-dimensional flux vector $F^{x}$ and the scalar flux $F^{\eta}$ are given by

$$
\begin{aligned}
F^{x}(x, \eta, t)=- & \sum_{K, K^{\prime} \in \mathcal{K}} a_{K}(x, \eta) C_{K K^{\prime}}^{-1}(x, \eta) \cdot \\
& \cdot\left(a_{K^{\prime}}(x, \eta)^{*} \mathrm{e}^{-V_{0}} \nabla_{x}\left(\mathrm{e}^{V_{0}} \frac{\rho}{N}\right)+A_{K^{\prime}}(x, \eta)^{*} \mathrm{e}^{-\eta} \partial_{\eta}\left(\mathrm{e}^{\eta} \frac{\rho}{N}\right)\right), \\
F^{\eta}(x, \eta, t)=- & \sum_{K, K^{\prime} \in \mathcal{K}} A_{K}(x, \eta)^{T} C_{K K^{\prime}}^{-1}(x, \eta) \cdot \\
& \cdot\left(a_{K^{\prime}}(x, \eta)^{*} \mathrm{e}^{-V_{0}} \nabla_{x}\left(\mathrm{e}^{V_{0}} \frac{\rho}{N}\right)+A_{K^{\prime}}(x, \eta)^{*} \mathrm{e}^{-\eta} \partial_{\eta}\left(\mathrm{e}^{\eta} \frac{\rho}{N}\right)\right) .
\end{aligned}
$$


Remarks.

- Without making any further assumptions on the confining potential $V_{1}$, no more specific information is available about the coefficients $a_{K}$ and $A_{K}$ in (4.19a) and (4.19b).

- Computationally, the actual challenge lies in computing the inconspicuous looking term $C_{K K^{\prime}}^{-1}(x, \eta)$ in (4.21). $K$ is a multiindex of the same dimension as the angular variable $\theta$, and $\theta$ denotes an angle in the $(6-2 d)$-dimensional $(y, w)$-space. Hence $K$ and $\theta$ have $5-2 d$ components.

For the case of a plate where $d=2, K$ is a scalar and the matrix $C(x, \eta)$ must be inverted for every point $(x, \eta)$.

For the case of a tube where $d=1$, the multiindex $K$ has three components and the resulting tensor $C(x, \eta)$ is very large even if a moderate number of expansions terms are used in each component of $\theta$.

- Computing $C_{K K^{\prime}}^{-1}(x, \eta)$ for a general confining potential $V_{1}$ still represents a reduction in computational complexity compared to solving the original Boltzmann equation, since the inversion of $C$ must be performed only once for a given structure.

- Since the idea of deriving large-time asymptotic approximations to the Boltzmann equation is to arrive at a relatively simple macroscopic system, we will treat, in the next section, the special case where the confinement potential $V_{1}$ is harmonic, i.e., where $V_{1}(x, y)$ is a quadratic function of the $(3-d)$ dimensional vector $y$ with general $x$-dependent coefficients.

Boundary conditions. So far we have not specified the boundary conditions for the conservation law (4.20). The boundary conditions in the $x$-direction depend on the application under consideration. Usually, they will consist of a combination of insulating walls (i.e., $\nu \cdot F^{x}=0$ where $\nu$ is the normal vector) and connections to a bath meaning that the density $\rho$ is prescribed by a given equilibrium bath density. The conservation law must hold for $\eta \in[0, \infty)$ and $F^{\eta}(x, 0, t)=0$ must hold because of mass conservation.

\section{Harmonic confinement potentials}

As will be seen in this section, the formulas (4.21) for the flux vectors $F^{x}$ and $F^{y}$ simplify dramatically in the case where the potential $V_{1}$ is quadratic in the variable $y$. Since the inversion of the matrix $C$ in (4.21) presents a major computational challenge only in the case of transport in a tube $(d=1)$, we will restrict ourselves to this case here. Hence, for the rest of this section, we will assume that $x, v \in \mathbb{R}$ and $y, w \in \mathbb{R}^{2}$. The coefficients $A_{K}$ in (4.21) are therefore scalar and the gradient $\nabla_{x}$ becomes a partial derivative $\partial_{x}$. In this case we assume that the confinement potential $V_{1}(x, y)$ is of the form

$$
V_{1}(x, y)=\frac{1}{2}(y-b(x))^{T} B(x)(y-b(x)),
$$

where $y, b \in \mathbb{R}^{2}$ and the diagonal matrix $B(x)$ is given by

$$
B(x)=\left(\begin{array}{cc}
B_{1}(x) & 0 \\
0 & B_{2}(x)
\end{array}\right)
$$

We require that $B_{1}(x)>0$ and $B_{2}(x)>0$ for all $x$ so that the particles are indeed confined. 
5.1. Constructing approximate harmonic potentials. Assuming that the confinement potential $V_{1}$ has the quadratic form (5.1) may seem slightly arbitrary at first. The physical relevance of the resulting model depends to a large extent on the quality of the approximate expression (5.1). However, this assumption will drastically simplify the resulting conservation law as seen below.

The immediate question is how to choose the coefficients $B_{j}$ and $b_{j}, j \in\{1,2\}$, for a given potential $V_{1}(x, y)$. The guiding principle is that the forces acting in the confinement direction $y$ on the particle due to the exact potential $V_{1}$ should be approximated optimally by the approximate expression (5.1). Therefore the coefficients in (5.1) should be chosen so that the resulting approximate field in the $y$-direction, which is of the form $B(x)(y-b(x))$, approximates the exact field $\nabla_{y} V_{1}$ locally for every value of $x$. Hence we choose the coefficients $B_{j}$ and $b_{j}, j \in\{1,2\}$, in (5.1) to locally minimize the functional

$$
\int_{\mathcal{B}}\left|B(x)(y-b(x))-\nabla_{y} V_{1}(x, y)\right|^{2} \mathrm{~d} y
$$

for every value of $x$. Here $\mathcal{B}$ denotes an appropriate domain in the $y$-space enclosing the structure. We choose to approximate the forces in the $L^{2}$-norm, since this yields a simple nonlinear least squares problem for every value of $x$. Thus the coefficients $B_{j}$ and $b_{j}, j \in\{1,2\}$, are computed from the equations

$$
\begin{aligned}
\int_{\mathcal{B}}\left(y_{j}-b_{j}\right)\left(B_{j}\left(y_{j}-b_{j}\right)-\partial_{y_{j}} V_{1}\right) \mathrm{d} y=0 & \forall j \in\{1,2\}, \\
\int_{\mathcal{B}} B_{j}\left(y_{j}-b_{j}\right)-\partial_{y_{j}} V_{1} \mathrm{~d} y=0 & \forall j \in\{1,2\}
\end{aligned}
$$

giving four-albeit nonlinear-equations for the four coefficient functions $B_{j}(x)$ and $b_{j}(x), j \in\{1,2\}$. If the potential $V_{1}(x, y)$ is given either in analytic or tabular form, the solution of the system (5.2) is straightforward.

5.2. The fluxes for harmonic confinement potentials. We define the variable transformation $(y, w)=\Omega(x, \eta, \theta)$ in two stages. First, we transform $\left(y_{j}, w_{j}\right)$, $j \in\{1,2\}$, using polar coordinates. We set

$$
y_{j}=: b_{j}+\sqrt{\frac{2 r_{j}}{B_{j}}} \cos \theta_{j}, \quad w_{j}=: \sqrt{2 r_{j}} \sin \theta_{j},
$$

with $r_{j} \in[0, \infty)$ and $\theta_{j} \in[-\pi, \pi)$ for $j \in\{1,2\}$. This gives the energy $\mathcal{E}_{y}$ as $\mathcal{E}_{y}(x, y, w)=$ $r_{1}+r_{2}$. Next, we use the transformation

$$
\eta:=r_{1}+r_{2}, \quad \theta_{3}:=\frac{r_{2}-r_{1}}{r_{2}+r_{1}}, \quad r_{1}=\eta \frac{1-\theta_{3}}{2}, \quad r_{2}=\eta \frac{1+\theta_{3}}{2},
$$

with $\eta \in[0, \infty)$ and $\theta_{3} \in[-1,1]$. Now the energy $\mathcal{E}_{y}$ is given by $\mathcal{E}_{y}(x, y, w)=\eta$. Combining the two transformations yields

$$
\left(\begin{array}{c}
y \\
w
\end{array}\right)=\Omega(x, \eta, \theta)=\left(\begin{array}{c}
\Omega_{y}(x, \eta, \theta) \\
\Omega_{w}(x, \eta, \theta)
\end{array}\right)=\left(\begin{array}{c}
b_{1}+\sqrt{\frac{\eta\left(1-\theta_{3}\right)}{B_{1}}} \cos \theta_{1} \\
b_{2}+\sqrt{\frac{\eta\left(1+\theta_{3}\right)}{B_{2}}} \cos \theta_{2} \\
\sqrt{\eta\left(1-\theta_{3}\right)} \sin \theta_{1} \\
\sqrt{\eta\left(1+\theta_{3}\right)} \sin \theta_{2}
\end{array}\right) .
$$


Transforming the volume element in the involved integrals according to the two transformations yields $\mathrm{d} y w=\frac{1}{\sqrt{B_{1} B_{2}}} \mathrm{~d} r_{1} r_{2} \theta_{1} \theta_{2}=\frac{\eta}{2 \sqrt{B_{1} B_{2}}} \mathrm{~d} \eta \theta_{1} \theta_{2} \theta_{3}$ and hence we find

$$
\sigma(x, \eta, \theta)=|\operatorname{det}(\partial \Omega(x, \eta, \theta))|=\frac{\eta}{2 \sqrt{B_{1}(x) B_{2}(x)}}
$$

in (4.19). For the density of states function $N$, we immediately obtain

$$
N(x, \eta)=\int \sigma(x, \eta, \theta) \mathrm{d} \theta=\frac{4 \pi^{2} \eta}{\sqrt{B_{1}(x) B_{2}(x)}} .
$$

The variable $\theta$ is a three-dimensional angle in the four-dimensional $(y, w)$-space and $\theta_{3}$ is the sine of the azimuthal angle varying in the interval $\left(-\frac{\pi}{2}, \frac{\pi}{2}\right)$. Therefore the natural choice for the basis functions in $\S 4.3$ is

$$
\kappa_{K}(\theta):=\frac{1}{2 \pi} \mathrm{e}^{\mathrm{i} k_{1} \theta_{1}+\mathrm{i} k_{2} \theta_{2}} L_{k_{3}}\left(\theta_{3}\right), \quad K=\left(k_{1}, k_{2}, k_{3}\right),
$$

where $L_{k_{3}}\left(\theta_{3}\right)$ the Legendre polynomial of degree $k_{3}$ normalized in the $L^{2}$-norm on the interval $[-1,1]$.

We now turn to computing the coefficients $a_{K}, A_{K}$, and $C_{K K^{\prime}}$ in (4.19). Using the fact that the zero-order basis function $\kappa_{0}:=\kappa_{(0,0,0)}$ is given by $\kappa_{0}(\theta)=1 /(2 \pi \sqrt{2})$, the coefficient $a_{K}$ in (4.19a) simplifies to

$$
a_{K}(x, \eta)=\int \kappa_{K} \sigma \mathrm{d} \theta=\sqrt{2} \pi \frac{\eta}{\sqrt{B_{1} B_{2}}} \int \kappa_{0}^{*} \kappa_{K} \mathrm{~d} \theta=\frac{\sqrt{2} \pi \eta}{\sqrt{B_{1} B_{2}}} \delta_{0, K}
$$

To compute the coefficient $A_{K}(x, \eta)$ in Equation (4.19b), we first compute the term $\partial_{x} V_{1}(x, y)=\sum_{j=1}^{2} \frac{1}{2} \partial_{x} B_{j}\left(y_{j}-b_{j}\right)^{2}-B_{j}\left(y-b_{j}\right) \partial_{x} b_{j}$. After the variable transformation it becomes

$$
\begin{aligned}
& \partial_{1} V_{1}\left(x, \Omega_{y}(x, y, \theta)\right) \\
= & \sum_{j=1}^{2}\left(\frac{1}{2} \partial_{x}\left(\ln B_{j}\right) \eta\left(1+(-1)^{j} \theta_{3}\right) \cos ^{2} \theta_{j}-\sqrt{B_{j} \eta\left(1+(-1)^{j} \theta_{3}\right)} \cos \theta_{j} \partial_{x} b_{j}\right),
\end{aligned}
$$

which yields the coefficient $A_{K}$ in $(4.19 \mathrm{~b})$ as

$$
\begin{aligned}
& A_{K}(x, \eta)=\frac{\eta}{4 \pi \sqrt{B_{1} B_{2}}} \int_{-1}^{1} \int_{-\pi}^{\pi} \int_{-\pi}^{\pi} \mathrm{e}^{\mathrm{i} k_{1} \theta_{1}+\mathrm{i} k_{2} \theta_{2}} L_{k_{3}}\left(\theta_{3}\right) . \\
& \cdot \sum_{j=1}^{2}\left(\frac{1}{2} \partial_{x}\left(\ln B_{j}\right) \eta\left(1+(-1)^{j} \theta_{3}\right) \cos ^{2} \theta_{j}-\sqrt{B_{j} \eta\left(1+(-1)^{j} \theta_{3}\right)} \cos \theta_{j} \partial_{x} b_{j}\right) \mathrm{d} \theta_{1} \theta_{2} \theta_{3} .
\end{aligned}
$$

After integrating and defining

$$
L_{k_{3}}^{ \pm}:=\int_{-1}^{1} \sqrt{1 \pm \theta_{3}} L_{k_{3}}\left(\theta_{3}\right) \mathrm{d} \theta_{3}
$$

we find

$$
\begin{aligned}
& A_{K}(x, \eta)=\frac{\pi \eta}{2 \sqrt{B_{1} B_{2}}}\left(\frac{1}{4} \eta \partial_{x}\left(\ln B_{1}\right) \delta_{k_{2}, 0}\left(\delta_{k_{1},-2}+2 \delta_{k_{1}, 0}+\delta_{k_{1}, 2}\right)\left(\sqrt{2} \delta_{k_{3}, 0}-\sqrt{2 / 3} \delta_{k_{3}, 1}\right)\right. \\
&+\frac{1}{4} \eta \partial_{x}\left(\ln B_{2}\right) \delta_{k_{1}, 0}\left(\delta_{k_{2},-2}+2 \delta_{k_{2}, 0}+\delta_{k_{2}, 2}\right)\left(\sqrt{2} \delta_{k_{3}, 0}+\sqrt{2 / 3} \delta_{k_{3}, 1}\right) \\
&\left.-\sqrt{\eta B_{1}} \partial_{x} b_{1} \delta_{k_{2}, 0}\left(\delta_{k_{1},-1}+\delta_{k_{1}, 1}\right) L_{k_{3}}^{-}-\sqrt{\eta B_{2}} \partial_{x} b_{2} \delta_{k_{1}, 0}\left(\delta_{k_{2},-1}+\delta_{k_{2}, 1}\right) L_{k_{3}}^{+}\right) .
\end{aligned}
$$


Explicit expressions for $L_{k_{3}}^{ \pm}$are given in Lemma D.1 in Appendix D.

To compute the matrix element $C_{K K^{\prime}}$ in (4.19c), we first have to compute the vector $S(x, \eta, \theta)=\left(\gamma_{21} \gamma_{12}^{T}-\gamma_{22} \gamma_{11}^{T}\right)(x, \Omega(x, \eta, \theta))$. Computing the matrix elements $\gamma_{j k}$ represents an elementary but quite tedious calculation. In the special case of a harmonic potential, it is actually easier to compute the vector $S$ by directly transforming the Poisson bracket. The equation $\left\{\mathcal{E}_{y}, \phi\right\}_{y w}=S^{T} \nabla_{\theta} \phi_{1}$ must hold for any function $\phi(y, w)=\phi_{1}(\eta, \theta)$. Given the shape (5.1) of the potential $V_{1}$, the equation

$$
\left\{\mathcal{E}_{y}, \phi\right\}_{y w}=\sum_{j=1}^{2}\left(w_{j} \partial_{y_{j}} \phi-B_{j}\left(y_{j}-b_{j}\right) \partial_{w_{j}} \phi\right)
$$

holds and straightforward calculus yields

$$
\left\{\mathcal{E}_{y}, \phi\right\}_{y w}=-\sum_{j=1}^{2} \sqrt{B_{j}} \partial_{\theta_{j}} \phi_{1}(\eta, \theta) .
$$

Therefore the equation $S(x, \eta, \theta)=-\left(\sqrt{B_{1}(x)}, \sqrt{B_{2}(x)}, 0\right)^{T}$ holds. Computing the matrix element $C_{K K^{\prime}}$ in $(4.19 \mathrm{c})$ yields

$$
\begin{aligned}
C_{K K^{\prime}}(x, \eta)=\frac{1}{8 \pi^{2}} & \frac{\eta}{\sqrt{B_{1} B_{2}}} \int \mathrm{e}^{-\mathrm{i} k_{1} \theta_{1}-\mathrm{i} k_{2} \theta_{2}} L_{k_{3}}\left(\theta_{3}\right) . \\
& \left.\cdot\left(\begin{array}{c}
\sqrt{B_{1}} \\
\sqrt{B_{2}} \\
0
\end{array}\right) \cdot\left(\begin{array}{c}
\mathrm{i} k_{1}^{\prime} L_{k_{3}^{\prime}}\left(\theta_{3}\right) \\
\mathrm{i} k_{2}^{\prime} L_{k_{3}^{\prime}}\left(\theta_{3}\right) \\
\partial_{\theta_{3}} L_{k_{3}^{\prime}}^{\prime}\left(\theta_{3}\right)
\end{array}\right)+\frac{1}{\tau} L_{k_{3}^{\prime}}\left(\theta_{3}\right)\right) \mathrm{e}^{\mathrm{i} k_{1}^{\prime} \theta_{1}+\mathrm{i} k_{2}^{\prime} \theta_{2}} \mathrm{~d} \theta \\
=\frac{1}{8 \pi^{2}} & \frac{\eta}{\sqrt{B_{1} B_{2}}} \int \mathrm{e}^{-\mathrm{i} k_{1} \theta_{1}-\mathrm{i} k_{2} \theta_{2}} L_{k_{3}}\left(\theta_{3}\right) \cdot \\
& \cdot\left(-\mathrm{i} k_{1}^{\prime} \sqrt{B_{1}}-\mathrm{i} k_{2}^{\prime} \sqrt{B_{2}}+\frac{1}{\tau}\right) \mathrm{e}^{\mathrm{i} k_{1}^{\prime} \theta_{1}+\mathrm{i} k_{2}^{\prime} \theta_{2}} L_{k_{3}^{\prime}}\left(\theta_{3}\right) \mathrm{d} \theta,
\end{aligned}
$$

and after integrating out $\theta$ we obtain

$$
C_{K K^{\prime}}(x, \eta)=\frac{\eta}{2 \sqrt{B_{1} B_{2}}}\left(\frac{1}{\tau}-\mathrm{i} k_{1} \sqrt{B_{1}}-\mathrm{i} k_{2} \sqrt{B_{2}}\right) \delta_{K K^{\prime}} .
$$

Thus the choice of a harmonic confinement potential makes the matrix $C(x, \eta)$ diagonal and the elements $C_{K K^{\prime}}^{-1}(x, \eta)$ of its inverse are given by

$$
C_{K K^{\prime}}^{-1}(x, \eta)=\frac{2 \tau \sqrt{B_{1} B_{2}}}{\eta} \frac{1+\tau\left(k_{1} \sqrt{B_{1}}+k_{2} \sqrt{B_{2}}\right) \mathrm{i}}{1+\tau^{2}\left(k_{1} \sqrt{B_{1}}+k_{2} \sqrt{B_{2}}\right)^{2}} \delta_{K K^{\prime}} .
$$

We note that the inverse always exists.

The formulas (5.5), (5.7), and (5.8) provide all the details to compute the expression for the fluxes in (4.21). It still remains to choose the index set $\mathcal{K}$, i.e., the number of terms used in the expansion (4.14) of $g_{1}(x, \eta, \theta, t)$. We note that because of (5.5) the term $a_{K}$ only gives a contribution for $K=0$. Also the coefficient $A_{K}$ in (5.7) vanishes for $\left|k_{1}\right|>2$ and $\left|k_{2}\right|>2$, since the complex exponentials are orthogonal to $\cos \theta_{j}$ and $\cos ^{2} \theta_{j}$ for all $j \in\{1,2\}$ in this case. Therefore, regardless of the number 
of terms used in the expansion, the fluxes $F^{x}$ and $F^{\eta}$ in (4.21) are of the form

$$
\begin{aligned}
& F^{x}(x, \eta, t)=-a_{0}(x, \eta) C_{00}^{-1}(x, \eta) \cdot \\
& \cdot\left(a_{0}(x, \eta)^{*} \mathrm{e}^{-V_{0}} \nabla_{x}\left(\mathrm{e}^{V_{0}} \frac{\rho}{N}\right)+A_{0}(x, \eta)^{*} \mathrm{e}^{-\eta} \partial_{\eta}\left(\mathrm{e}^{\eta} \frac{\rho}{N}\right)\right), \\
& F^{\eta}(x, \eta, t)=-\sum_{k_{1}=-2}^{2} \sum_{k_{2}=-2}^{2} \sum_{k_{3}=0}^{\infty} A_{K}(x, \eta) C_{K K}^{-1}(x, \eta) . \\
& \cdot\left(\delta_{0, K} a_{0}(x, \eta)^{*} \mathrm{e}^{-V_{0}} \nabla_{x}\left(\mathrm{e}^{V_{0}} \frac{\rho}{N}\right)+A_{K}(x, \eta)^{*} \mathrm{e}^{-\eta} \partial_{\eta}\left(\mathrm{e}^{\eta} \frac{\rho}{N}\right)\right) .
\end{aligned}
$$

Therefore we can use all terms in $\theta_{1}$ and $\theta_{2}$ in the expansion (4.14) of $g_{1}(x, \eta, \theta, t)$ and choose the index sets of both $k_{1}$ and $k_{2}$ as $\mathbb{Z}$. This means that in the case of a harmonic confinement potential $V_{1}$ the components of the initial density $f(x, y, v, w, t=$ 0 ) corresponding to the higher modes in $\theta_{1}$ and $\theta_{2}$ do not contribute to the large-time dynamics after the projection in $\S 3$.

We proceed to calculate explicit expressions for the fluxes. To simplify notation, we define $T_{1}:=\mathrm{e}^{-V_{0}} \nabla_{x}\left(\mathrm{e}^{V_{0}} \frac{\rho}{N}\right)$ and $T_{2}:=\mathrm{e}^{-\eta} \partial_{\eta}\left(\mathrm{e}^{\eta} \frac{\rho}{N}\right)$. We find

$$
F^{x}(x, \eta, t)=-\frac{4 \pi^{2} \tau \eta}{\sqrt{B_{1} B_{2}}} T_{1}-\frac{\pi^{2} \tau \eta^{2}}{\sqrt{B_{1} B_{2}}}\left(\partial_{x}\left(\ln B_{1}\right)+\partial_{x}\left(\ln B_{2}\right)\right) T_{2}
$$

and

$$
\begin{aligned}
& F^{\eta}(x, \eta, t)=-\frac{\pi^{2} \tau \eta^{2}}{\sqrt{B_{1} B_{2}}}\left(\partial_{x}\left(\ln B_{1}\right)+\partial_{x}\left(\ln B_{2}\right)\right) T_{1} \\
&-\sum_{k_{3}=0}^{\infty} \frac{\pi^{2} \tau \eta^{2}}{24 \sqrt{B_{1} B_{2}}}\left(4 \eta \partial_{x}\left(\ln B_{1}\right) \partial_{x}\left(\ln B_{2}\right)\left(3 \delta_{k_{3}, 0}-\delta_{k_{3}, 1}\right)\right. \\
&+\left(\frac{\eta\left(3+8 \tau^{2} B_{1}\right)\left(\partial_{x}\left(\ln B_{1}\right)\right)^{2}}{1+4 \tau^{2} B_{1}}+\frac{\eta\left(3+8 \tau^{2} B_{2}\right)\left(\partial_{x}\left(\ln B_{2}\right)\right)^{2}}{1+4 \tau^{2} B_{2}}\right)\left(3 \delta_{k_{3}, 0}+\delta_{k_{3}, 1}\right) \\
&\left.\quad+\frac{24 B_{1}\left(\partial_{x} b_{1}\right)^{2}}{1+\tau^{2} B_{1}}\left(L_{k_{3}}^{-}\right)^{2}+\frac{24 B_{2}\left(\partial_{x} b_{2}\right)^{2}}{1+\tau^{2} B_{2}}\left(L_{k_{3}}^{+}\right)^{2}\right) T_{2} .
\end{aligned}
$$

Using the expressions for $L_{k_{3}}^{ \pm}$in Lemma D.1, the sums over $\left(L_{k_{3}}^{ \pm}\right)^{2}$ become

$$
\sum_{k_{3}=0}^{K_{3}}\left(L_{k_{3}}^{+}\right)^{2}=\sum_{k_{3}=0}^{K_{3}}\left(L_{k_{3}}^{-}\right)^{2}=\frac{16\left(2 K_{3}^{4}+8 K_{3}^{3}+11 K_{3}^{2}+6 K_{3}+1\right)}{\left(4 K_{3}^{2}+8 K_{3}+3\right)^{2}}
$$

and clearly their limit as $K_{3} \rightarrow \infty$ is 2 . Therefore we can choose the index set of $k_{3}$ as $\mathbb{Z}_{0}^{+}$and the whole index set as $\mathcal{K}:=\mathbb{Z} \times \mathbb{Z} \times \mathbb{Z}_{0}^{+}$. Finally we obtain

$$
\begin{aligned}
F^{\eta}(x, \eta, t)=- & \frac{\pi^{2} \tau \eta^{2}}{\sqrt{B_{1} B_{2}}}\left(\partial_{x}\left(\ln B_{1}\right)+\partial_{x}\left(\ln B_{2}\right)\right) T_{1} \\
- & \frac{\pi^{2} \tau \eta^{2}}{6 \sqrt{B_{1} B_{2}}}\left(\frac{12 B_{1}\left(\partial_{x} b_{1}\right)^{2}}{1+\tau^{2} B_{1}}+\frac{12 B_{2}\left(\partial_{x} b_{2}\right)^{2}}{1+\tau^{2} B_{2}}+2 \eta \partial_{x}\left(\ln B_{1}\right) \partial_{x}\left(\ln B_{2}\right)\right. \\
& \left.\quad+\frac{\eta\left(3+8 \tau^{2} B_{1}\right)\left(\partial_{x}\left(\ln B_{1}\right)\right)^{2}}{1+4 \tau^{2} B_{1}}+\frac{\eta\left(3+8 \tau^{2} B_{2}\right)\left(\partial_{x}\left(\ln B_{2}\right)\right)^{2}}{1+4 \tau^{2} B_{2}}\right) T_{2} .
\end{aligned}
$$


Equations (5.10) and (5.11) give the constitutive relations for the fluxes used in the conservation law

$$
\partial_{t} \rho(x, \eta, t)+\partial_{x} F^{x}(x, \eta, t)+\partial_{\eta} F^{\eta}(x, \eta, t)=0
$$

in the case of a tube with a harmonic confinement potential after inserting the expressions for $a_{k}, A_{K}$, and $C_{K K}^{-1}$ from (5.5), (5.7), and (5.8). The resulting equation is a diffusion equation because of the first-order partial derivatives with respect to $x$ and $\eta$ in the definition of the fluxes (5.9). The resulting diffusion matrix has automatically the right eigenvalues, since the entropy estimate (4.16) holds.

All the coefficients of the derivatives in the fluxes are indeed real numbers. Importantly, the three infinite sums over $k_{1}, k_{2}$, and $k_{3}$ have been reduced to explicit expressions. In summary, numerical solutions of this problem can be calculated as fast as solutions of the two-dimensional drift-diffusion equations.

\section{Numerical results}

Using the explicit form of the transport coefficients, solutions of the diffusion system can be calculated very efficiently. A finite-volume scheme was implemented for the case of tubes $(d=1)$. The numerical examples presented in the following illustrate the features of the solutions due to confinement.

6.1. First example. We consider an example with no applied potential first. The simulation domain is defined by $x \in[0,10]$ and $\eta \in[0,10]$. We set $V_{0}(x):=0, \tau:=1$, $B_{1}(x):=B_{2}(x):=1+5 \exp \left(-(x-5)^{2} / 2\right)$, and $b_{1}(x):=b_{2}(x):=0$. The boundary conditions are the Maxwellian distributions $\rho(0, \eta):=2 \exp (-\eta)$ and $\rho(10, \eta):=\exp (-\eta)$; this means that the concentration gradient between the two baths drives the current.

The resulting concentration $\rho$ and fluxes $F^{x}$ and $F^{\eta}$ are shown in Figure 6.1. The influence of the boundary conditions and the reduction in concentration due to confinement in the middle of the channel at $x=5$ is seen in the concentration $\rho$. The streamlines plot for the fluxes shows that the particles are pushed towards higher energies because of the confinement potential and some of the particles are reflected back to the left boundary.

To illustrate the convergence properties as the grid becomes finer, the values for the current $\int F^{x}(10, \eta) \mathrm{d} \eta$ are noted here since it is important in applications: for a grid spacing of $\Delta x:=\Delta \eta:=1$ the current is 0.048108 ; for $1 / 2$ it is 0.057327 , for $1 / 4$ it is 0.061520 , for $1 / 8$ it is 0.062836 , for $1 / 16$ it is 0.063117 , and for $1 / 32$ it is 0.063120 .

6.2. Second example. In the second example, we apply a potential $V_{0}(x):=x$ and leave the rest of the parameters unchanged. This potential drives particles from the right to the left in opposite direction to the concentration gradient of the baths.

The resulting concentration $\rho$ and fluxes $F^{x}$ and $F^{\eta}$ are shown in Figure 6.2. Again it is observed that the confinement potential drives the particles towards higher energies in the middle of the channel at $x=5$. Additionally, we see in the plot of the concentration that the influx of particles from the right boundary due to the strong applied potential and the confinement in the middle result in a pile-up of particles at around $x=7$.

The current values for different grid sizes are the following: for a grid spacing of 1 the current is -0.932237 , for $1 / 2$ it is -0.916781 , for $1 / 4$ it is -0.905318 , for $1 / 8$ it is -0.897971 , for $1 / 16$ it is -0.893613 , and for $1 / 32$ it is -0.891181 . Due to the applied potential, the current has opposite sign now and it is much larger. 

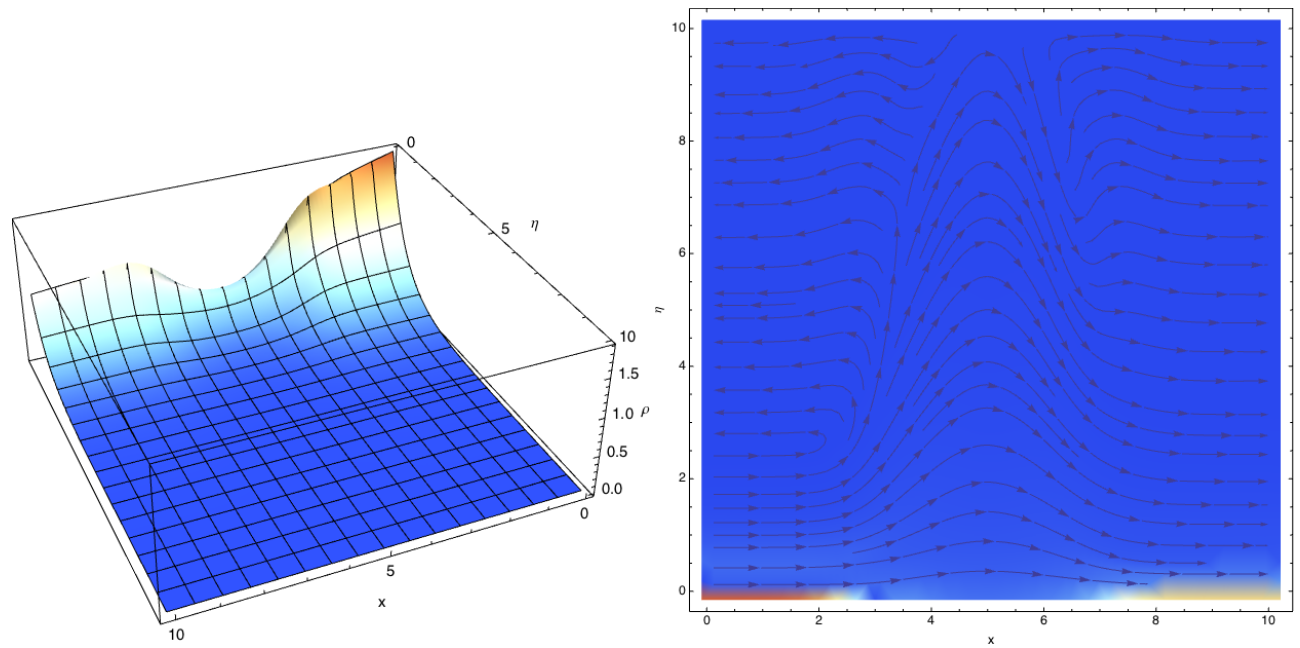

Fig. 6.1. The concentration $\rho(x, \eta)$ (left) and a streamlines plot of the flux $F^{x}(x, \eta) \mathbf{e}_{x}+$ $F^{\eta}(x, \eta) \mathbf{e}_{\eta}$ (right). Large values are red, small values blue.
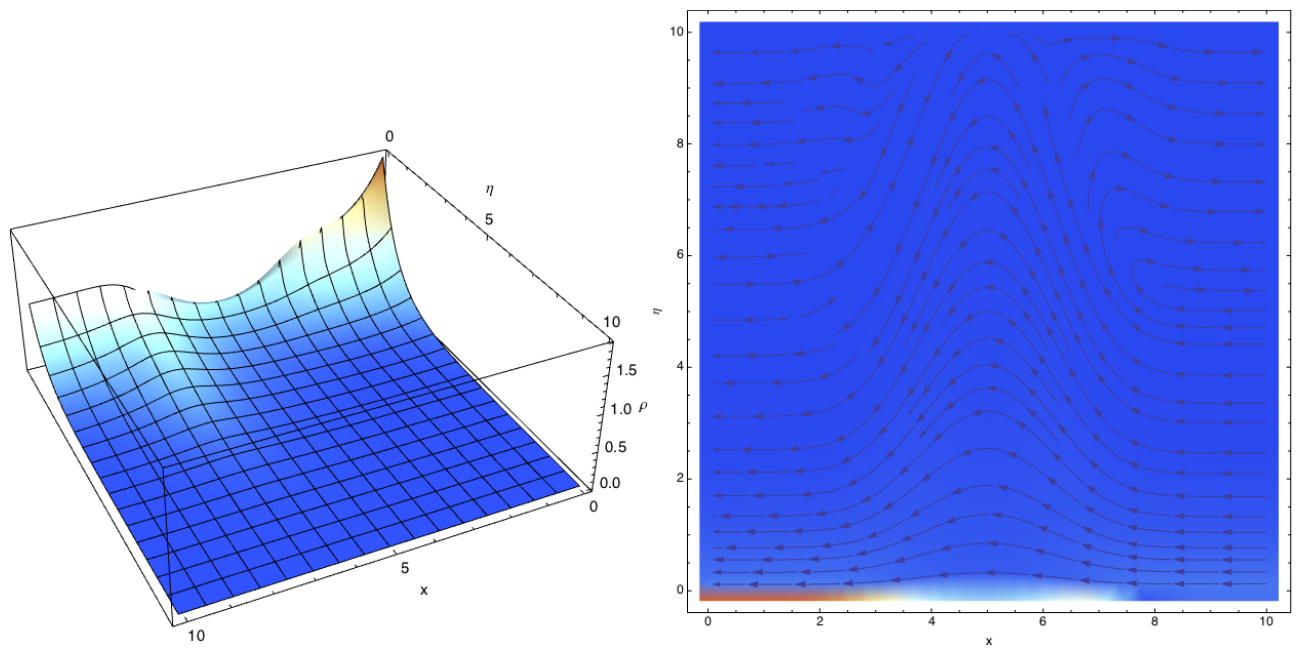

FIG. 6.2. The concentration $\rho(x, \eta)$ (left) and a streamlines plot of the flux $F^{x}(x, \eta) \mathbf{e}_{x}+$ $F^{\eta}(x, \eta) \mathbf{e}_{\eta}$ (right). Large values are red, small values blue.

\section{Conclusion}

We have derived a diffusion-type equation for particle transport in confined structures from the Boltzmann transport equation. In the case of confinement in cylindrical structures or pipes, the six independent spatial and momentum variables in the Boltzmann transport equation are reduced to two independent variables, namely position in the longitudinal spatial direction and energy. The Galerkin approximation and the variable transformation are chosen so that the geometry dependent transport coefficients can be given explicitly and therefore the diffusion-type equation can be solved efficiently. Entropy estimates are given for solutions of the diffusion equation. 
A numerical scheme for the resulting diffusion equation was implemented. The responses of the numerical solutions to changes in the parameters, the confinement potential, and the boundary conditions are consistent with the physics of the underlying transport problem.

\section{Acknowledgment}

The authors acknowledge discussions with Naoufel Ben Abdallah.

\section{Appendix A. Proof of Lemma 3.1.}

Proof. To prove Equation (3.11), we have to show that the identity

$$
-\int f(x, y, v, w, t)^{*} \mathcal{L}_{2}[\rho] \mathrm{d} x y v w=c \int \mathcal{L}_{1}\left[\mathrm{e}^{-\mathcal{E}_{x}-\mathcal{E}_{y}} f\right](x, \eta)^{*} \frac{\mathrm{e}^{V_{0}(x)+\eta} \rho(x, \eta, t)}{N(x, \eta)} \mathrm{d} x \eta
$$

holds for all functions $f(x, y, v, w, t)$ and $\rho(x, \eta, t)$. Inserting for $\mathcal{L}_{1}$ yields

$$
\begin{aligned}
& c \int \mathcal{L}_{1}\left[\mathrm{e}^{-\mathcal{E}_{x}-\mathcal{E}_{y}} f\right]^{*} \frac{\mathrm{e}^{V_{0}(x)+\eta} \rho(x, \eta, t)}{N(x, \eta)} \mathrm{d} x \eta \\
= & c \int\left\{\mathcal{E}_{x}+\mathcal{E}_{y}, \mathrm{e}^{-\mathcal{E}_{x}-\mathcal{E}_{y}} f^{*}\right\}_{x v} \frac{\mathrm{e}^{V_{0}(x)+\mathcal{E}_{y}} \rho\left(x, \mathcal{E}_{y}, t\right)}{N\left(x, \mathcal{E}_{y}\right)} \mathrm{d} x y v w .
\end{aligned}
$$

Since the Poisson bracket obeys the product rule of differentiation, we find

$$
\begin{aligned}
\left\{\mathcal{E}_{x}+\mathcal{E}_{y}, \mathrm{e}^{-\mathcal{E}_{x}-\mathcal{E}_{y}} f^{*}\right\}_{x v} & =\left\{\mathcal{E}_{x}+\mathcal{E}_{y}, f^{*}\right\}_{x v} \mathrm{e}^{-\mathcal{E}_{x}-\mathcal{E}_{y}}+\left\{\mathcal{E}_{x}+\mathcal{E}_{y}, \mathrm{e}^{-\mathcal{E}_{x}-\mathcal{E}_{y}}\right\}_{x v} f^{*} \\
& =\left\{\mathcal{E}_{x}+\mathcal{E}_{y}, f^{*}\right\}_{x v} \mathrm{e}^{-\mathcal{E}_{x}-\mathcal{E}_{y}}
\end{aligned}
$$

due to the identity $\left\{\mathcal{E}_{x}+\mathcal{E}_{y}, \mathrm{e}^{-\mathcal{E}_{x}-\mathcal{E}_{y}}\right\}_{x v}=0$. Hence we have

$$
\begin{aligned}
& c \int \mathcal{L}_{1}\left[\mathrm{e}^{-\mathcal{E}_{x}-\mathcal{E}_{y}} f\right]^{*} \frac{\mathrm{e}^{V_{0}(x)+\eta} \rho(x, \eta, t)}{N(x, \eta)} \mathrm{d} x \eta \\
= & c \int\left\{\mathcal{E}_{x}+\mathcal{E}_{y}, f^{*}\right\}_{x v} \frac{\mathrm{e}^{V_{0}(x)-\mathcal{E}_{x}} \rho\left(x, \mathcal{E}_{y}, t\right)}{N\left(x, \mathcal{E}_{y}\right)} \mathrm{d} x y v w .
\end{aligned}
$$

Using the identity $c \mathrm{e}^{V_{0}(x)-\mathcal{E}_{x}}=M(v)$ and the cyclicity of the commutator under the trace, this can be written as

$$
\begin{aligned}
& c \int \mathcal{L}_{1}\left[\mathrm{e}^{-\mathcal{E}_{x}-\mathcal{E}_{y}} f\right]^{*} \frac{\mathrm{e}^{V_{0}(x)+\eta} \rho(x, \eta, t)}{N(x, \eta)} \mathrm{d} x \eta \\
&=\int f^{*}\left\{\frac{M(v)}{N\left(x, \mathcal{E}_{y}\right)} \rho\left(x, \mathcal{E}_{y}, t\right), \mathcal{E}_{x}+\mathcal{E}_{y}\right\}_{x v} \mathrm{~d} x y v w=-\int f(x, y, v, w, t)^{*} \mathcal{L}_{2}[\rho] \mathrm{d} x y v w
\end{aligned}
$$

yielding (A.1) and consequently (3.11). To show (3.12), we set and calculate

$$
\begin{aligned}
I:=\int \mathrm{e}^{\mathcal{E}_{x}+\mathcal{E}_{y}} f^{*}\left\{\mathcal{E}_{y}, f\right\}_{y w} \mathrm{~d} y w=\int\left\{\mathrm{e}^{\mathcal{E}_{x}+\mathcal{E}_{y}} f^{*}, \mathcal{E}_{y}\right\}_{y w} f \mathrm{~d} y w \\
=\int \mathrm{e}^{\mathcal{E}_{x}+\mathcal{E}_{y}}\left\{f^{*}, \mathcal{E}_{y}\right\}_{y w} f \mathrm{~d} y w+\int f^{*}\left\{\mathrm{e}^{\mathcal{E}_{x}+\mathcal{E}_{y}}, \mathcal{E}_{y}\right\}_{y w} f \mathrm{~d} y w \\
=\int \mathrm{e}^{\mathcal{E}_{x}+\mathcal{E}_{y}} f\left\{f^{*}, \mathcal{E}_{y}\right\}_{y w} \mathrm{~d} y w=-\int \mathrm{e}^{\mathcal{E}_{x}+\mathcal{E}_{y}} f\left\{\mathcal{E}_{y}, f\right\}_{y w}^{*} \mathrm{~d} y w=-I^{*},
\end{aligned}
$$


which implies $\mathfrak{R e}(I)=0$.

Appendix B. Proof of Lemma 4.1.

Proof. We start with the observation that, since the Maxwellian $M$ is of the form $M(v)=c \mathrm{e}^{-|v|^{2} / 2}$ with $\int M(v) \mathrm{d} v=1$, integration by parts yields the matrix identity $\int v^{T} v M(v) \mathrm{d} v=I$, where $I$ is the $d \times d$ identity matrix. Inserting the definition of $\mathcal{L}_{1}$ into (4.1a) and integrating against a test function $\phi(x)$ gives

$$
\int \phi(x) \Lambda_{1}[g](x, \eta, t) \mathrm{d} x=\int \phi(x) \delta\left(\mathcal{E}_{y}-\eta\right)\left\{\mathcal{E}_{x}+\mathcal{E}_{y}, M(v) v \cdot g\right\}_{x v} \mathrm{~d} x y v w .
$$

Using the cyclicity of the commutator under the trace yields

$$
\begin{gathered}
\int \phi(x) \Lambda_{1}[g](x, \eta, t) \mathrm{d} x=\int\left\{\phi(x) \delta\left(\mathcal{E}_{y}-\eta\right), \mathcal{E}_{x}+\mathcal{E}_{y}\right\}_{x v} M(v) v \cdot g \mathrm{~d} x y v w \\
=\int\left(\nabla_{v}\left(\phi(x) \delta\left(\mathcal{E}_{y}(x, y, w)-\eta\right)\right) \cdot \nabla_{x}\left(\mathcal{E}_{x}+\mathcal{E}_{y}\right)-\nabla_{x}\left(\phi(x) \delta\left(\mathcal{E}_{y}-\eta\right)\right) \cdot \nabla_{v}\left(\mathcal{E}_{x}+\mathcal{E}_{y}\right)\right) . \\
\cdot M(v) v \cdot g \mathrm{~d} x y v w \\
=\int\left(\left(-\delta\left(\mathcal{E}_{y}-\eta\right) \nabla_{x} \phi(x)-\phi(x) \delta^{\prime}\left(\mathcal{E}_{y}-\eta\right) \nabla_{x} V_{1}\right) \cdot v\right) M(v) v \cdot g \mathrm{~d} x y v w .
\end{gathered}
$$

Then, using the identity $\int v^{T} v M(v) \mathrm{d} v=I$, we obtain

$$
\begin{aligned}
\int \phi(x) \Lambda_{1}[g](x, \eta, t) \mathrm{d} x & =\int\left(-\delta\left(\mathcal{E}_{y}-\eta\right) \nabla_{x} \phi(x)-\phi(x) \delta^{\prime}\left(\mathcal{E}_{y}-\eta\right) \nabla_{x} V_{1}\right) \cdot g \mathrm{~d} x y w \\
& =\int \phi(x)\left(\nabla_{x} \cdot\left(\delta\left(\mathcal{E}_{y}-\eta\right) g\right)+\partial_{\eta}\left(\delta\left(\mathcal{E}_{y}-\eta\right) \nabla_{x} V_{1} \cdot g\right)\right) \mathrm{d} x y w,
\end{aligned}
$$

which proves Equation (4.3).

The relation (4.4) is essentially the same as the adjoint relation (3.11) in Lemma 3.1: Equation (3.11) implies that

$$
\int f^{*} \mathcal{L}_{2}[\rho] \mathrm{d} x y v w=-c \int \mathcal{L}_{1}\left[\mathrm{e}^{-\mathcal{E}_{x}-\mathcal{E}_{y}} f\right]^{*} \frac{\mathrm{e}^{V_{0}+\eta} \rho(x, \eta, t)}{N(x, \eta)} \mathrm{d} x \eta
$$

holds for all functions $f(x, y, v, w, t)$ and $\rho(x, \eta, t)$. Using the definition (4.1) of $\Lambda_{1}$ and $\Lambda_{2}$ and setting $f(x, y, v, w, t):=\mathrm{e}^{\mathcal{E}_{x}+\mathcal{E}_{y}} M(v) v \cdot g(x, y, w, t)$ gives

$$
\int \mathrm{e}^{\mathcal{E}_{x}+\mathcal{E}_{y}} M(v)^{2} g^{H} v\left(v \cdot \Lambda_{2}[\rho]\right) \mathrm{d} x y v w=-c \int \Lambda_{1}[g]^{*} \frac{\mathrm{e}^{V_{0}+\eta} \rho(x, \eta, t)}{N(x, \eta)} \mathrm{d} x \eta .
$$

Using the identities $\mathrm{e}^{\mathcal{E}_{x}} M(v)=c \mathrm{e}^{V_{0}}$ and $\int v^{T} v M(v) \mathrm{d} v=I$ yields

$$
c \int \mathrm{e}^{V_{0}+\mathcal{E}_{y}} g^{H} \Lambda_{2}[\rho] \mathrm{d} x y w=-c \int \Lambda_{1}[g]^{*} \frac{\mathrm{e}^{V_{0}+\eta} \rho}{N} \mathrm{~d} x \eta
$$

and therefore

$$
\mathrm{e}^{V_{0}+\mathcal{E}_{y}} \Lambda_{2}[\rho]=-\Lambda_{1}^{\text {adj }}\left[\frac{\mathrm{e}^{V_{0}+\eta} \rho}{N}\right]
$$

holds, which proves Equation (4.4). Finally, the relation (4.5) is essentially the same as Equation (3.12) in Lemma 3.1 formulated for vector valued functions $g$. 


\section{Appendix C. Proof of Lemma 4.3.}

Proof. For any function $\phi(x, y, w)$ and its transform $\phi_{1}(x, u, \theta)=$ $\phi_{1}(x, \Gamma(x, y, w)):=\phi(x, y, w)$, their gradients are related by

$$
\nabla_{y} \phi^{T}=\partial_{u} \phi_{1}^{T} \gamma_{11}+\nabla_{\theta} \phi_{1}^{T} \gamma_{21}, \quad \nabla_{w} \phi^{T}=\partial_{u} \phi_{1}^{T} \gamma_{12}+\nabla_{\theta} \phi_{1}^{T} \gamma_{22} .
$$

Differentiating the identity $\mathcal{E}_{y}(x, \Omega(u, \theta))=u$ with respect to $u$ and $\theta$ gives, using the chain rule, the relations $\nabla_{y} \mathcal{E}_{y}^{T} \omega_{11}+\nabla_{w} \mathcal{E}_{y}^{T} \omega_{21}=1$ and $\nabla_{y} \mathcal{E}_{y}^{T} \omega_{12}+\nabla_{w} \mathcal{E}_{y}^{T} \omega_{22}=0$ or, in matrix form,

$$
\left(\nabla_{y} \mathcal{E}_{y}^{T}, \nabla_{w} \mathcal{E}_{y}^{T}\right) \partial \Omega=(1,0) .
$$

Multiplying with $\partial \Gamma=\partial \Omega^{-1}$ from the right gives $\left(\nabla_{y} \mathcal{E}_{y}^{T}, \nabla_{w} \mathcal{E}_{y}^{T}\right)=\left(\gamma_{11}, \gamma_{12}\right)$ and hence we have

$$
\gamma_{11}(x, y, w)=\nabla_{y} \mathcal{E}_{y}(x, y, w)^{T}, \quad \gamma_{12}(x, y, w)=\nabla_{w} \mathcal{E}_{y}(x, y, w)^{T} .
$$

Computing the (scalar) commutator as

$$
\begin{aligned}
\left\{\mathcal{E}_{y}, \phi\right\}_{y w}(x, y, w)=\nabla_{w} \mathcal{E}_{y}^{T} \nabla_{y} \phi-\nabla_{y} \mathcal{E}_{y}^{T} \nabla_{w} \phi & =\gamma_{12} \nabla_{y} \phi-\gamma_{11} \nabla_{w} \phi \\
& =\nabla_{y} \phi^{T} \gamma_{12}^{T}-\nabla_{w} \phi^{T} \gamma_{11}^{T}
\end{aligned}
$$

and using the transformation (C.1) of the gradients of $\phi$ yields

$$
\left\{\mathcal{E}_{y}, \phi\right\}_{y w}(x, y, w)=\partial_{u} \phi_{1}(x, \Gamma)\left(\gamma_{11} \gamma_{12}^{T}-\gamma_{12} \gamma_{11}^{T}\right)+\nabla_{\theta} \phi_{1}(x, \Gamma)^{T}\left(\gamma_{21} \gamma_{12}^{T}-\gamma_{22} \gamma_{11}^{T}\right) .
$$

Considering the dimensions of the matrix elements $\gamma_{j k}$, i.e., $\gamma_{11}$ and $\gamma_{12}$ are both $1 \times(3-d)$-dimensional matrices and $\gamma_{11} \gamma_{12}^{T}$ is a scalar, we find that $\gamma_{11} \gamma_{12}^{T}-\gamma_{12} \gamma_{11}^{T}=0$ holds. Thus we have

$$
\begin{aligned}
\left\{\mathcal{E}_{y}, \phi\right\}_{y w}(x, y, w) & =\nabla_{\theta} \phi_{1}(x, \Gamma(x, y, w))^{T} S(x, \Gamma(x, y, w)) \\
= & \left(S(x, \Gamma(x, y, w)) \cdot \nabla_{\theta}\right) \phi_{1}(x, \Gamma(x, y, w)) .
\end{aligned}
$$

The relation (4.12) is obtained directly from (4.5) in Lemma 4.1 by performing the appropriate variable substitution in the integral. This concludes the proof.

\section{Appendix D. The integrals $L_{n}^{ \pm}$.}

Here we prove explicit formulas for the integrals $L_{n}^{ \pm}$defined in (5.6).

Lemma D.1. Let $L_{n}$ be the Legendre polynomials on the interval $[-1,1]$ normalized in the $L^{2}$-norm. Then the equation

$$
L_{n}^{ \pm}=\int_{-1}^{1} \sqrt{1 \pm x} L_{n}(x) \mathrm{d} x=\frac{-4(\mp 1)^{n}}{(2 n+3)(2 n-1) \sqrt{2 n+1}} \quad \forall n \in \mathbb{N}
$$

holds.

Proof. We denote the non-normalized Legendre polynomials with $P_{n}(1)=1$ by $P_{n}$. Since $\left\|L_{n}\right\|=1$ and $\left\|P_{n}\right\|^{2}=2 /(2 n+1)$,

$$
L_{n}=\sqrt{\frac{2 n+1}{2}} P_{n}
$$


holds. It is well-known that the Legendre polynomials $P_{n}$ can be written as

$$
P_{n}(x)=\frac{1}{2^{n} n !} \partial_{x}^{n}\left(x^{2}-1\right)^{n}
$$

using Rodrigues' formula. This expression yields

$$
\begin{aligned}
L_{n}^{ \pm} & =\frac{1}{2^{n} n !} \sqrt{\frac{2 n+1}{2}} \int_{-1}^{1} \sqrt{1 \pm x} \partial_{x}^{n}\left(x^{2}-1\right)^{n} \mathrm{~d} x \\
& =\frac{1}{2^{n} n !} \sqrt{\frac{2 n+1}{2}}(\mp 1)^{n}\left[\frac{1}{2}\right]_{n} \int_{-1}^{1}(1 \pm x)^{-n+1 / 2}\left(x^{2}-1\right)^{n} \mathrm{~d} x
\end{aligned}
$$

after $n$ partial integrations. Here $[a]_{n}$ denotes the falling sequential product

$$
[a]_{n}:=\prod_{j=0}^{n-1}(a-j) .
$$

It is straightforward to see that the boundary terms vanish; the derivatives of $\left(x^{2}-1\right)^{n}$ vanish at \pm 1 , since they all contain a factor $x^{2}-1$. The last integral can be written as a beta function after substituting $y:=(1 \pm x) / 2$, yielding

$$
\begin{aligned}
\int_{-1}^{1}(1 \pm x)^{-n+1 / 2}\left(x^{2}-1\right)^{n} \mathrm{~d} x=( \pm 1)^{n} \int_{-1}^{1} \sqrt{1 \pm x}(x \mp 1)^{n} \mathrm{~d} x \\
=2 \sqrt{2}(-2)^{n} B(3 / 2, n+1)=2 \sqrt{2}(-2)^{n} \frac{\Gamma(3 / 2) \Gamma(n+1)}{\Gamma(n+5 / 2)}=\frac{\sqrt{2}(-2)^{n} n !}{[n+3 / 2]_{n+2}},
\end{aligned}
$$

since $\Gamma(n+5 / 2)=[n+3 / 2]_{n+2} \sqrt{\pi}$. In summary, we obtain

$$
\begin{aligned}
L_{n}^{ \pm}=( \pm 1)^{n} \sqrt{2 n+1} \frac{[1 / 2]_{n}}{[n+3 / 2]_{n+2}} & =( \pm 1)^{n} \sqrt{2 n+1} \frac{(-1)^{n-1}[n-3 / 2]_{n-1}(-1 / 2)}{[n+3 / 2]_{n+2}} \\
& =(\mp 1)^{n} \sqrt{2 n+1} \frac{1}{(n+3 / 2)(n+1 / 2)(n-1 / 2)},
\end{aligned}
$$

which concludes the proof.

\section{REFERENCES}

[1] N. Ben Abdallah, P. Degond, P. Markowich, and C. Schmeiser, High field approximations of the spherical harmonics expansion model for semiconductors, Z. Angew. Math. Phys., 52, 201-230, 2001.

[2] N. Ben Abdallah, F. Méhats, C. Schmeiser, and R. Weisshäupl, The nonlinear Schrödinger equation with a strongly anisotropic harmonic potential, SIAM J. Math. Anal., 37, 189199, 2005.

[3] N. Ben Abdallah, F. Méhats, and N. Vauchelet, Diffusive transport of partially quantized particles: Existence, uniqueness and long-time behaviour, Proc. Edinburgh Math. Soc., (2), 49, 513-549, 2006

[4] B. Corry, S. Kuyucak, and S. Chung, Tests of continuum theories as models of ion channels. II: Poisson-Nernst-Planck theory versus Brownian dynamics, Biophys. J., 78, 2000.

[5] M. Fischetti and S. Laux, Monte Carlo study of electron transport in silicon inversion layers, Phys. Rev. B, 48, 2244-2274, 1993.

[6] C. Heitzinger, Y. Liu, N. J. Mauser, C. Ringhofer, and R. W. Dutton, Calculation of fluctuations in boundary layers of nanowire field-effect biosensors, J. Comput. Theor. Nanosci., 7, 2574-2580, 2010. 
[7] C. Heitzinger, N. Mauser, and C. Ringhofer, Multiscale modeling of planar and nanowire fieldeffect biosensors, SIAM J. Appl. Math., 70, 1634-1654, 2010.

[8] C. Heitzinger and C. Ringhofer, Multiscale modeling of fluctuations in stochastic elliptic PDE models of nanosensors, SIAM J. Appl. Math., submitted.

[9] Y. Liu, K. Lilja, C. Heitzinger, and R. W. Dutton, Overcoming the screening-induced performance limits of nanowire biosensors: A simulation study on the effect of electro-diffusion flow, in IEDM 2008 Technical Digest, San Francisco, CA, USA, Dec., 491-494, 2008.

[10] W. Nonner, L. Catacuzzeno, and R. Eisenberg, Binding and selectivity in L-type calcium channels: A mean spherical approximation, Biophys. J., 79, 1976-1992, 2000.

[11] F. Rossi and T. Kuhn, Theory of ultrafast phenomena in photoexcited semiconductors, Rev. Modern Phys., 74, 895-950, 2002.

[12] B. Roux and M. Karplus, Ion transport in a model gramicidin channel, Biophys. J., 59, 961-981, 1991.

[13] E. Stern, J. Klemic, D. Routenberg, P. Wyrembak, D. Turner-Evans, A. Hamilton, D. LaVan, T. Fahmy, and M. Reed, Label-free immunodetection with CMOS-compatible semiconducting nanowires, Nature, 445, 519-522, 2007.

[14] E. Stern, A. Vacic, N. Rajan, J. Criscione, J. Park, B. Ilic, D. Mooney, M. Reed, and T. Fahmy, Label-free biomarker detection from whole blood, Nature Nanotechnology, 5, 138-142, 2010.

[15] G. Zheng, F. Patolsky, Y. Cui, W. U. Wang, and C. M. Lieber, Multiplexed electrical detection of cancer markers with nanowire sensor arrays, Nature Biotechnology, 23, 1294-1301, 2005. 